\title{
La cuestión de la idoneidad de los vídeos educativos de matemáticas: una experiencia de análisis con futuros maestros de educación primaria
}

\section{The issue of didactical suitability in mathematics educational videos: experience of analysis with prospective primary school teachers}

\author{
Dra. María BURGOS. Profesora Contratada Doctora. Universidad de Granada (mariaburgos@ugr.es). \\ Dr. Pablo BELTRÁN-PELLICER. Profesor Asociado. Universidad de Zaragoza (pbeltran@unizar.es). \\ Dr. Juan D. GODINO. Catedrático. Universidad de Granada (jgodino@ugr.es).
}

\section{Resumen:}

La cantidad de vídeos educativos disponibles en Internet sobre los más variados temas está aumentando a un ritmo acelerado. Así, nos encontramos vídeos de matemáticas que cubren prácticamente cualquier tópico curricular, aunque su calidad como recurso didáctico es muy desigual. En consecuencia, es necesario proporcionar a los profesores herramientas que les permitan analizar la pertinencia del uso de estos vídeos, teniendo en cuenta los diversos aspectos implicados. En este trabajo se describe el diseño, implementación y resultados de una acción formativa con 93 futuros maestros de educación primaria, orientada al desarrollo de la competencia de análisis de la idoneidad didáctica de vídeos sobre proporcionalidad. El análisis a priori del vídeo reveló errores e imprecisiones significativas en las definiciones, proposiciones y procedimientos, así como carencias o inexactitudes en los argumentos, por lo que el nivel de idoneidad epistémica se valora como media. Sin embargo, la mayoría de los futuros docentes valoraron su grado de idoneidad como alto en casi todos los componentes. Los estudiantes consideran positivo el estudio de la idoneidad didáctica y su implementación a través de

Trabajo realizado en el marco del proyecto de investigación EDU2016-74848-P (AEI, FEDER) y Grupos FQM-126 (Junta de Andalucía) y S36_17D (Gobierno de Aragón y Fondo Social Europeo), España.

Fecha de recepción de la versión definitiva de este artículo: 25-11-2019.

Cómo citar este artículo: Burgos, M., Beltrán-Pellicer, P. y Godino, J. D. (2020). La cuestión de la idoneidad de los vídeos educativos de matemáticas: una experiencia de análisis con futuros maestros de educación primaria| The issue of didactical suitability in mathematics educational videos: experience of analysis with prospective primary school teachers. Revista Española de Pedagogía, 78 (275), 27-49. doi: https://doi.org/10.22550/REP78-1-2020-07 
componentes e indicadores, considerando que esta actividad facilita la reflexión profesional. No obstante, el dominio de esta herramienta requiere incrementar el número y variedad de vídeos para analizar y mayor discusión colectiva de los resultados de los análisis que realizan los estudiantes.

Descriptores: formación de profesores, enfoque ontosemiótico, idoneidad didáctica, vídeos educativos, proporcionalidad.

\section{Abstract:}

The number of educational videos available on the internet on the most varied topics is rapidly increasing. These include mathematics videos that cover virtually any type of curriculum content. However, their quality as a learning resource varies greatly. As a result, it is necessary to provide teachers with tools to enable them to analyse the appropriateness of using educational videos, considering the various aspects involved. This paper describes the design, implementation and results of an educational intervention with 93 prospective primary school teachers, focussed on developing their ability to analyse the educational suitability of videos about proportionality. Preliminary analysis of the video revealed significant errors and inaccuracies in the definitions, propositions, and procedures, as well as shortcomings and inaccuracies in the arguments, and so its level of epistemic suitability is rated as medium. However, the majority of the prospective teachers rated its degree of suitability as high in almost all components. Students regard studying didactic suitability and implementing it through components and indicators as positive, believing that this activity facilitates professional reflection. However, mastering this tool requires analysing a greater number and variety of videos and further collective discussion of the results of the analyses performed by the students.

Keywords: teachers' education, onto-semiotic approach, didactical suitability, educational videos, proportionality.

\section{Introducción}

El uso de vídeos educativos disponibles en YouTube y otras plataformas ha crecido de forma desorbitada en los últimos años, convirtiéndose en un prometedor recurso de aprendizaje para los estudiantes y el público en general (Azer, AlGrain, AlKhelaif, y AlEshaiwi, 2013).

Estos recursos educativos y los modelos pedagógicos que los usan, como la clase invertida (flipped learning) (Bergmann y
Sams, 2012), deben ser un tema de investigación educativa ya que no está claro cómo es posible lograr un aprendizaje significativo mediante el visionado de clases grabadas. De hecho, diversos investigadores discuten el papel que el uso de YouTube y otros medios sociales puede jugar en la educación formal, analizando cómo se organizan los recursos online y cómo pueden ser insertados como herramientas informales en contextos educativos precisos (Borba, Askar, Engelbrecht, Gadanidis, Llinares y 
Aguilar, 2016; Dabbagh y Kitsantas, 2012; Duffy, 2008; Portugal, Arruda y Passos, 2018; Ramírez, 2010). Se considera necesario que desde la didáctica se indague sobre la adecuación de los recursos educativos en línea, asegurando que la tecnología esté en concordancia con los objetivos de aprendizaje (Turney, Robinson, Lee y Soutar, 2009).

Las investigaciones en didáctica de las matemáticas sobre la utilización de vídeos educativos señalan la importancia de que los propios docentes valoren y recomienden los vídeos idóneos para su alumnado (Beltrán-Pellicer, Giacomone y Burgos, 2018; Ruiz-Reyes, Contreras, Arteaga y Oviedo, 2017; Santos, 2018), dado que algunos de ellos muestran procedimientos formalmente incorrectos, no todos indican el nivel educativo al que se dirigen, o los significados puestos en juego pueden no ser pertinentes con lo que se está tratando en clase. En este sentido, resulta clara la necesidad de diseñar e implementar experiencias formativas que permitan promover el crecimiento profesional y el desarrollo de conocimientos y competencias en el profesorado (Chapman, 2014; English, 2008; Mason, 2016; Ponte y Chapman, 2016; Sadler, 2013).

Dentro de esta problemática, el objetivo de la presente investigación es el diseño, implementación y evaluación de una acción formativa con futuros maestros de educación primaria, focalizada en el desarrollo de conocimientos y competencia para el análisis de la idoneidad epistémica de vídeos educativos sobre proporcionalidad disponibles en Internet.

El trabajo se estructura en los siguientes apartados. En la sección 2 se describe el marco teórico y el problema específico de investigación. La sección 3 describe el diseño del proceso formativo experimentado. En la sección 4 se incluye el análisis a priori del vídeo sobre proporcionalidad que es usado como instrumento de evaluación de las competencias logradas por los futuros profesores. La sección 5 muestra detalladamente los resultados de la experiencia, analizando de manera cualitativa y cuantitativa los informes elaborados de manera individual por los futuros profesores. La última sección incluye la síntesis, implicaciones y limitaciones de la investigación.

\section{Marco teórico y problema de investigación}

En el campo de investigación sobre formación de profesores de matemáticas se están usando diferentes marcos teóricos para categorizar y promover los diferentes tipos de conocimientos y competencias profesionales (Pino-Fan y Godino, 2015). Consideramos que el modelo de Conocimientos y competencias didáctico-matemáticas (CCDM) (Godino, Giacomone, Batanero y Font, 2017; Breda, Pino-Fan y Font, 2017), elaborado en el marco del Enfoque ontosemiótico del conocimiento y la instrucción matemáticos (EOS) (Godino, Batanero y Font, 2007), proporciona herramientas pertinentes para abordar nuestro problema de investigación. Este modelo resalta la importancia de diseñar e implementar recursos formativos que promuevan la competencia de análisis de la idoneidad didáctica por parte de los profesores. La noción de idoneidad didáctica se entiende como el grado en que un proceso de instrucción reúne ciertas características que permiten calificarlo 
como óptimo 0 adecuado, siendo el principal criterio la adaptación entre los significados personales construidos por los alumnos (aprendizaje) y los significados institucionales, ya sean pretendidos o implementados (enseñanza), considerando la influencia del entorno (Godino, 2013). Esto supone la articulación coherente y sistémica de seis criterios relativos a las facetas que intervienen en un proceso de instrucción (Godino et al., 2007): epistémica, ecológica, cognitiva, afectiva, interaccional y mediacional.

Se considera que un proceso de instrucción matemática tiene mayor idoneidad epistémica en la medida en que los significados institucionales implementados (o pretendidos) representan bien a un significado de referencia. El significado de referencia será relativo al nivel educativo correspondiente y deberá ser elaborado teniendo en cuenta los diversos tipos de problemas y contextos de uso del contenido objeto de enseñanza, así como las prácticas operativas y discursivas requeridas (Godino, 2013). Así, será necesario tener en cuenta el grado de adecuación de las situaciones-problemas, pero también será necesario prestar atención a la diversidad y adecuación de las representaciones, definiciones, procedimientos y proposiciones, así como a los argumentos que las sustentan. Una alta idoneidad desde el punto de vista epistémico requiere que las situaciones-problema propuestas involucren diversas representaciones, permitan a los estudiantes diversas maneras de abordarlas y requieran que los estudiantes interpreten, generalicen y justifiquen las soluciones. Además, los diversos significados parciales de los objetos matemáticos que aparecen involucrados de- ben estar conectados y articulados (Godino, Font, Wilhelmi y Lurduy, 2011).

El uso de la idoneidad didáctica permite al profesor hacer una reflexión sistemática sobre su propia práctica (Aroza, Godino y Beltrán-Pellicer, 2016; Beltrán-Pellicer, Godino y Giacomone, 2018; Posadas y Godino, 2017), pero también se puede aplicar para analizar aspectos parciales de los procesos instruccionales, como el uso de recursos tecnológicos. De manera específica, Beltrán-Pellicer, Giacomone y Burgos (2018) apoyándose en las herramientas teórico-metodológicas del EOS, analizaron el grado de idoneidad epistémica de una selección de los vídeos educativos más vistos por los usuarios en YouTube ${ }^{\mathrm{TM}}$ en relación con problemas de reparto proporcional. Encontraron, por un lado, una gran diversidad de enfoques y métodos de resolución, lo que puede interferir con el proceso de enseñanza y aprendizaje en el aula si estos vídeos no han sido previamente seleccionados o grabados por el docente. Por otro lado, la idoneidad epistémica de la muestra de vídeos analizados era muy desigual, con vídeos con errores e imprecisiones, además de que muchos de ellos ofrecían un tratamiento poco representativo 0 articulado del contenido matemático. Finalmente, observaron que los vídeos con métricas de mayor popularidad no coinciden con los más idóneos. Este trabajo constituye el principal antecedente de nuestra investigación.

Consideramos que sería deseable que los profesores conozcan la herramienta idoneidad didáctica y adquieran competencia para su uso en el análisis crítico de los recursos educativos, particularmente del uso de vídeos disponibles en Internet. 
Por otra parte, diversas investigaciones señalan que tanto los profesores en formación inicial como en servicio presentan dificultades para enseñar conceptos relacionados con la proporcionalidad (Bartell, Webel, Bowen y Dyson, 2013; Ben-Chaim, Keret e Ilany, 2012; Berk, Taber, Gorowara y Poetzl, 2009; Hilton y Hilton, 2018). Se hace preciso que la formación de profesores tenga en cuenta el desarrollo de conocimientos y competencias didáctico-matemáticas con relación a este tema, diseñando e implementado intervenciones formativas específicas. Esta es la razón por la cual el tema del vídeo, cuyo análisis se propone a los profesores en formación, es la proporcionalidad.

\section{Diseño del proceso formativo}

La experiencia formativa se realizó en el marco de la asignatura de Diseño y Desarrollo del Currículum en Educación Primaria durante el año lectivo 2018-2019, con noventa y tres estudiantes de tercer curso del Grado de Educación Primaria.

Durante los estudios de grado, los futuros profesores han recibido formación específica sobre aspectos epistémicos (contenido matemático), cognitivos (aprendizaje matemático, errores y dificultades), instruccionales y curriculares, de forma que en el momento en que se desarrolla la experiencia, los estudiantes deben ser capaces de poner en práctica el conocimiento adquirido para analizar, diseñar, fundamentar procesos de enseñanza-aprendizaje de acuerdo a unos contenidos específicos (en nuestro caso la proporcionalidad).
Además, de forma previa al desarrollo de esta investigación, y de acuerdo con el modelo CCDM asumido, se habían llevado a cabo talleres de formación con el grupo de estudiantes focalizados en el desarrollo de la competencia de análisis de significados globales (basado en la identificación de situaciones-problemas y prácticas operativas, discursivas y normativas implicadas en su resolución), y el análisis ontosemiótico de las prácticas (descripción de la trama de objetos y procesos implicados en las prácticas) que se ponen en juego en la actividad matemática de solución de problemas que involucran la proporcionalidad.

En la primera sesión se desarrolló un taller de dos horas de duración en el que se presentaron las características de la teoría de idoneidad didáctica y cómo se articulan entre sí las distintas dimensiones epistémica, cognitiva, afectiva, interaccional, mediacional y ecológica de un proceso de estudio determinado. Se pretende involucrar a los futuros maestros en una reflexión sobre la necesidad de disponer de un sistema de indicadores específicos que permitan valorar la práctica docente de manera sistemática.

En la siguiente sesión, también de dos horas de duración, los futuros maestros debían trabajar en equipos para analizar la idoneidad epistémica de vídeos educativos en línea, sobre proporcionalidad. El trabajo inicial en grupo permite a los estudiantes contrastar, discutir y enriquecer sus propuestas de valoración de idoneidad epistémica de distintos vídeos educativos en relación con la proporcionalidad. 
En la tercera fase, los estudiantes realizaron de manera individual las tareas que se describen en la siguiente sección como instrumento de evaluación final y cuyos resultados analizamos en este trabajo.

\section{Análisis a priori de la idonei- dad epistémica del vídeo}

En este apartado realizamos el análisis de la idoneidad epistémica, esto es, del conocimiento matemático que se pone en juego en el vídeo, que servirá de referencia para interpretar las respuestas dadas por los estudiantes a la valoración de la idoneidad del vídeo educativo. El análisis y valoración $a$ priori fue realizada de forma independiente por los investigadores y confrontada después para decidir una valoración común.

El vídeo educativo que se analiza ${ }^{1}$ trata el tema de la proporcionalidad directa desde el punto de vista aritmético presentando las nociones de razón y proporción (BenChaim, Keret e Ilany, 2012). Se trata de que los futuros maestros vean detenidamente el vídeo y decidan, de manera crítica y siguiendo los componentes e indicadores de idoneidad epistémica (Godino et al., 2007; Godino, 2013), su grado de idoneidad.
En general, las situaciones-problema propuestas en el vídeo aparecen contextualizadas y las ideas matemáticas están conectadas. Se proponen diversas maneras para abordar los problemas, pero consideramos que la muestra de dichos problemas no es suficientemente representativa ni articulada. Los métodos de solución que propone se aplican únicamente a problemas de valor perdido, en los que a priori se asume la condición de regularidad, y no se consideran, por ejemplo, problemas de comparación de razones. El vídeo cuenta con una gran riqueza de registros y representaciones lingüísticas: registros natural (tanto oral como escrito), simbólico (numérico o algebraico), tabular y gráfico.

Por otro lado, hemos identificado algunos errores e imprecisiones en la presentación de las reglas (definiciones y proposiciones) y los argumentos:

- Error de expresión en el tratamiento de las simplificaciones de las fracciones. Para simplificar se tachan los números que aparecen en el numerador y denominador dejando como superíndice los factores que van quedando al cancelar términos.

\section{GRÁFICo 1. Captura de pantalla del minuto 7.}

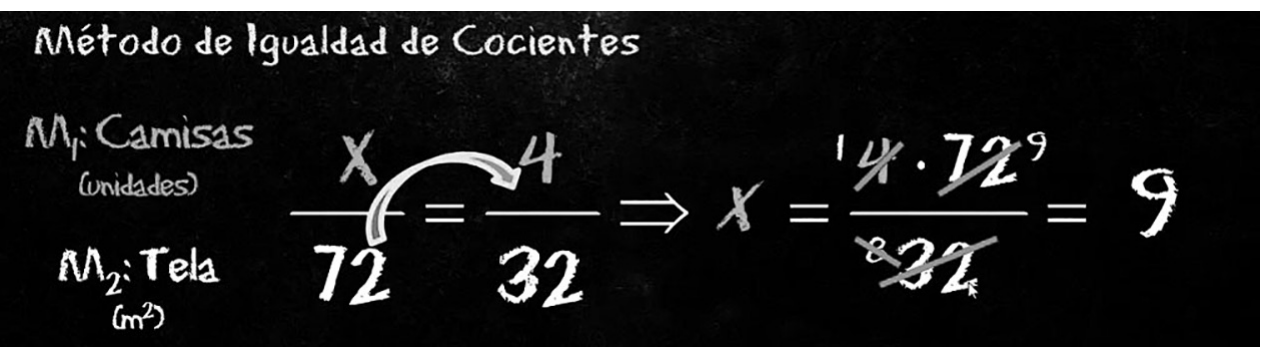

Fuente: Clasemáticas, 2012. 
- Error en la definición de razón y proporción.

(1:00) «La razón, matemáticamente hablando, quiere decir cociente. La proporción, la razón, el cociente entre esas dos magnitudes siempre es el mismo».

Aquí observamos un uso incorrecto de los conceptos de razón y proporción. Una razón no siempre es un cociente y no es lo mismo razón que proporción, confusión encontrada con alguna frecuencia en la práctica de la enseñanza.

- La regla de a doble, doble no tiene por qué denotar positivamente una relación de proporcionalidad directa.

(3:20) Presenta un «truco» para saber si dos magnitudes son directamente proporcionales: «Para saber si dos magnitudes guardan proporción directa basta comprobar que se cumple que al doble de una le corresponde el doble de la otra, al triple el triple».

Esta es una condición necesaria pero no suficiente para que dos magnitudes sean directamente proporcionales. La proporcionalidad de magnitudes es una función lineal establecida entre las cantidades de dichas magnitudes.

- Cuando se justifica la relación de proporcionalidad directa se basa en la rela- ción, al doble de una magnitud corresponde el doble de la otra.

(4:30) A continuación, indica a los alumnos la pregunta que deben formularse después de localizar las magnitudes: «iAl doble de camisas necesitaré el doble de tela?»

(4:43) «Si la respuesta es sí, que naturalmente en este vídeo va a ser sí, porque si no, nos estaríamos dedicando a otro tipo de proporcionalidad [...] estamos ante un problema de proporción directa».

Esto es incorrecto. La respuesta a una pregunta como la que formula puede ser no y que no aparezca involucrada ninguna otra situación de proporcionalidad.

- No se argumentan las operaciones en una proporción ni el porqué de la multiplicación en cruz.

Consideramos que el grado de idoneidad respecto a las relaciones entre objetos, en una escala ordinal de baja, media, alta, es media, ya que no todas las proposiciones y procedimientos tienen un argumento asociado. Además, los diversos significados de los objetos que intervienen en las prácticas aparecen identificados en algunas ocasiones, pero no siempre, por lo que también en este aspecto el grado de idoneidad es media.

GRÁfICO 2. Captura de pantalla en el minuto 8:15. Método general.

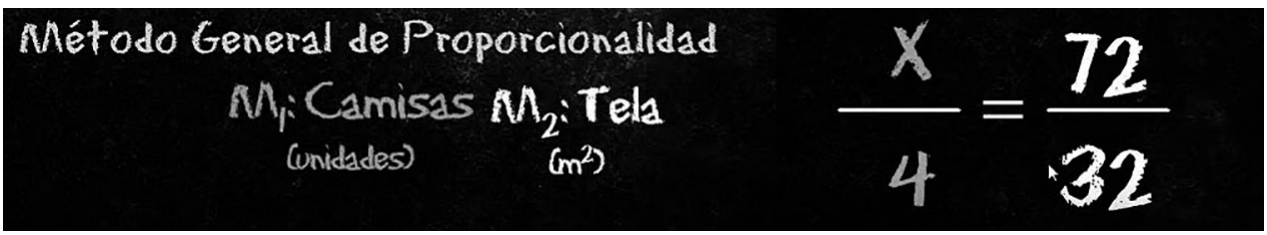

Fuente: Clasemáticas, 2012. 
(8:15) «Es decir, está la igualdad de cocientes entre las magnitudes, dentro de ellas mismas o comparándola con la otra magnitud, luego este cociente también es cierto».

En el primer método que presenta, el de «igualdad de cocientes», utiliza razones externas. Aquí, en el que el autor llama «método general de proporcionalidad», emplea razones internas, sin argumentar apropiadamente la relación entre ambas proporciones. Además, emplea el término proporción en lugar de razón para referirse a las fracciones que aparecen en la pantalla.

El último procedimiento para resolver problemas de proporcionalidad que presenta el autor del vídeo es la regla de tres.

(11:35) «Las magnitudes se colocan en forma de columna: ponemos x es a 72 como 4 camisas es a $32 »$.

GRÁfico 3. Captura de pantalla en el minuto 11:47. Método de la regla de tres.

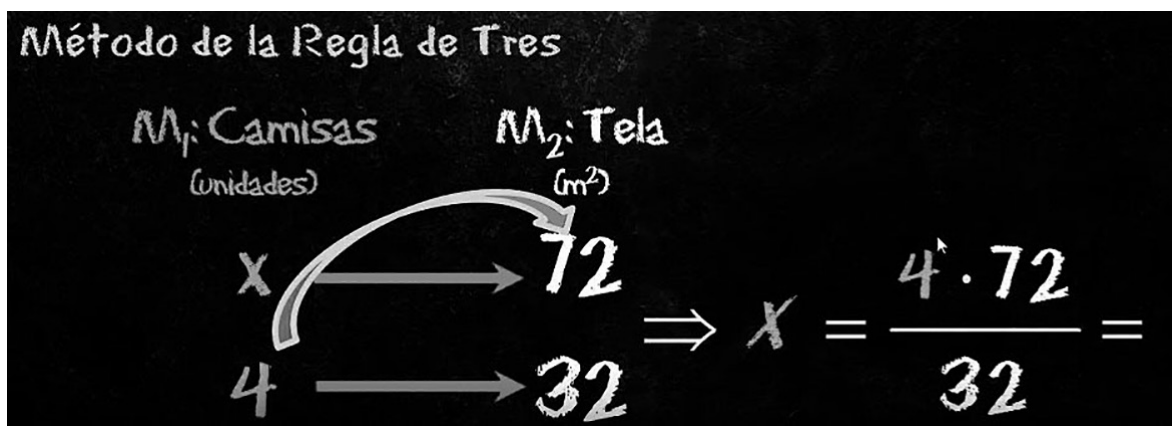

Fuente: Clasemáticas, 2012.

(11:47) «Y esto en realidad, si pudierais echar el vídeo hacia atrás, es el método general, lo que pasa es que no sé por qué os encanta esto de poner las flechas. El método general tiene una argumentación matemática y este no tanta».

Relaciona el método de regla de tres, que luego resuelve por multiplicación en cruz, con el método general. Se refiere a una «regla de tres degenerada», es decir, no plantea la ecuación proporcional, distinguiendo ese como otro método. Por lo tanto, lo que denomina como «regla de tres» es la disposición diagramática (me- diante flechas) acompañada de la multiplicación en cruz, que no aparece justificada en el vídeo.

En función de estos análisis se valora cuantitativamente el grado de idoneidad epistémica del vídeo en cada uno de los seis componentes, puntuándose cada indicador según su contribución a la idoneidad sea baja, media 0 alta $(0,1 \quad 02$ puntos, respectivamente). La puntuación total máxima será, por tanto, de 12 . En la Tabla 1 figuran las puntuaciones asignadas por el equipo investigador. 
La cuestión de la idoneidad de los vídeos educativos de matemáticas: una experiencia de ...

TABLA 1. Valoración de la idoneidad epistémica según componentes.

\begin{tabular}{|c|c|}
\hline Componente & Puntuación asignada al vídeo \\
\hline Situaciones-problema & 1 \\
\hline Lenguajes & 2 \\
\hline Reglas & 1 \\
\hline Argumentos & 1 \\
\hline Relaciones entre objetos & 1 \\
\hline Articulación de significados & 1 \\
\hline Puntuación final & 7 \\
\hline
\end{tabular}

Fuente: Elaboración propia.

\section{Resultados}

Para la segunda sesión de trabajo, se propuso a los estudiantes que, en primer lugar, vieran en casa tres vídeos ${ }^{2}$ sobre repartos directamente proporcionales con distinto grado de idoneidad. De manera individual debían decidir el mayor o menor grado de idoneidad de los mismos, así como su nivel de algebrización, teniendo en cuenta el tipo de solución desarrollada. Después, en clase, debían discutir con el grupo de trabajo sus valoraciones, elaborando después una propuesta grupal sobre el grado de idoneidad de los distintos vídeos. Las consignas dadas a los estudiantes para analizar los vídeos son las mismas que se plantean en la tarea final. Es decir, se tienen en cuenta: a) la variedad de situaciones-problemas propuestos; b) presencia de distintos registros de representación; c) claridad y corrección de las definiciones, proposiciones y procedimientos; d) argumentación de las proposiciones y procedimientos.

Como resultado de esta sesión, observamos que los futuros maestros pasaban por alto los errores en definiciones, proposiciones o procedimientos presentes en los vídeos y que en la puesta en común en su grupo de trabajo resultaba difícil consensuar el grado de idoneidad de los distintos vídeos. Discutir las propuestas individuales les llevó en muchos casos a modificar su análisis a priori, identificando nuevos elementos en el análisis que habían pasado desapercibidos.

En esta sección analizamos las respuestas dadas por los estudiantes a la tarea de evaluación final (tercera sesión de trabajo) y que reflejan, por consiguiente, los aprendizajes logrados por dichos estudiantes. Después, se analizan las respuestas dadas a la consigna de valoración cuantitativa en cada uno de los seis componentes de la idoneidad, así como la adecuación global del vídeo.

En la Tabla 2 se indican las frecuencias y porcentajes de las respuestas dadas sobre las características de las situaciones-problemas presentadas en el vídeo. 
TABLA 2. Variedad de situaciones-problemas propuestos ( $\mathrm{n}=93)$.

\begin{tabular}{|c|c|}
\hline Características de las situaciones & Frecuencias (\%) \\
\hline Se presenta una muestra representativa y articulada de problemas & $83(89.25)$ \\
\hline Las situaciones aparecen contextualizadas & $83(89.25)$ \\
\hline Las ideas matemáticas están conectadas & $87(93.55)$ \\
\hline Se proponen diversas maneras de abordar los problemas & $89(95.70)$ \\
\hline
\end{tabular}

Fuente: Elaboración propia.

Observamos que de forma mayoritaria los estudiantes reconocieron adecuadamente que en el vídeo educativo analizado las situaciones aparecen contextualizadas, las ideas están conectadas y se incluyen diversas formas de abordar los problemas. También en su mayoría (89.25\%) aceptaron que se presenta una muestra representativa y articulada de problemas.
Con relación a la presencia de distintas representaciones y registros de lenguaje, los estudiantes no tienen dificultad para identificar el registro natural y el registro simbólico. Sin embargo, identifican en menor grado el tabular y el gráfico. Otros estudiantes, en menor medida, identifican animación y otros tipos distintos (Tabla 3).

TABLA 3. Identificación de representaciones lingüísticas (n=93).

\begin{tabular}{|c|c|}
\hline Tipo de lenguaje & Frecuencias (\%) \\
\hline Natural (oral) & $92(98.9)$ \\
\hline Natural (escrito) & $93(100.0)$ \\
\hline Simbólico (numérico) & $89(95.7)$ \\
\hline Simbólico (algebraico) & $83(89.2)$ \\
\hline Tabular & $74(79.6)$ \\
\hline Gráfico & $28(30.1)$ \\
\hline Animación & $49(52.7)$ \\
\hline Otros (icónico, multimedia, diagramático, ...) & $10(10.8)$ \\
\hline
\end{tabular}

Fuente: Elaboración propia.

La mayoría de los futuros maestros tienen dificultades para localizar los errores 0 imprecisiones en las definiciones, proposiciones o procedimientos, así como en las argumentaciones dadas a las transformaciones, o cuando se justifica la relación de proporcionalidad directa en las situaciones planteadas (Tabla 4). 
TABLA 4. Claridad y corrección de definiciones, proposiciones, procedimientos y argumentos.

\begin{tabular}{|l|l|c|c|c|c|}
\hline \multirow{2}{*}{$\begin{array}{l}\text { Errores e imprecisiones en las } \\
\text { reglas y en los argumentos }\end{array}$} & No indica & $\begin{array}{l}\text { Dice explíci- } \\
\text { tamente que } \\
\text { no hay }\end{array}$ & $\begin{array}{l}\text { Sí pero no } \\
\text { especifica } \\
\text { cual }\end{array}$ & $\begin{array}{l}\text { Sí y } \\
\text { describe } \\
\text { cual }\end{array}$ \\
\cline { 2 - 6 } & $\begin{array}{l}\text { Errores de trata- } \\
\text { miento aritmético- } \\
\text { algebraico }\end{array}$ & $63(67.7)$ & $21(22.6)$ & $2(2.2)$ & $7(7.5)$ \\
\cline { 2 - 6 } Reglas & $\begin{array}{l}\text { Errores en defini- } \\
\text { ciones }\end{array}$ & $51(54.8)$ & $22(23.7)$ & $2(2.2)$ & $18(19.4)$ \\
\cline { 2 - 6 } & $\begin{array}{l}\text { Errores en propo- } \\
\text { siciones o procedi- } \\
\text { mientos }\end{array}$ & $51(54.8)$ & $21(22.6)$ & $5(5.4)$ & $16(17.2)$ \\
\hline \multirow{2}{*}{ Argumentos } & $\begin{array}{l}\text { Error o imprecisión } \\
\text { al argumentar una } \\
\text { relación de propor- } \\
\text { cionalidad. }\end{array}$ & $45(48.4)$ & $20(21.5)$ & $6(6.5)$ & $22(23.7)$ \\
\cline { 2 - 6 } & $\begin{array}{l}\text { Error o imprecisión } \\
\text { al argumentar una } \\
\text { transformación } \\
\text { aritmética-algebraica }\end{array}$ & $54(58.1)$ & $20(21.5)$ & $4(4.3)$ & $15(16.1)$ \\
\hline $\begin{array}{l}\text { Otros errores o im- } \\
\text { precisiones }\end{array}$ & $52(55.9)$ & $20(21.5)$ & $2(2.2)$ & $19(20.4)$ \\
\hline
\end{tabular}

Fuente: Elaboración propia.

Cuando los futuros maestros identifican errores de tratamiento aritmético 0 algebraico, algunos mencionan que el autor del vídeo no explica algunos de los símbolos usados, entre ellos los subíndices, o que simplificar los resultados de las operaciones puede confundir a los alumnos.

Con respecto a errores 0 imprecisiones en las definiciones que son reconocidos por los estudiantes, encontramos las siguientes categorías de respuestas:

- Error en la definición de razón. Esta es la categoría con mayor número de respuestas que identifican conflictos en las definiciones.
Por ejemplo, E50 menciona: «Razón: lo define como la proporción del cociente entre ambas magnitudes. Puede crear confusión, una alternativa seria "vínculo entre dos magnitudes que se pueden comparar entre sí"».

- Imprecisión en la definición de proporcionalidad directa.

- Definiciones confusas o no adaptadas al nivel de primaria.

- Además de estas categorías, algunos estudiantes incluyen como errores en las definiciones, apreciaciones no pertinentes con relación a procedimientos 
0 modos de expresión. Así, E84 refiere: «Puede causar confusión los nombres de los métodos empleados para realizar la proporción, ya que en distintas partes se pueden utilizar diferentes nombres para dichos métodos».

Los futuros maestros muestran dificultades para asignar los errores en la categoría adecuada y para hacer una descripción de forma pertinente. Hacen referencia a «explicaciones confusas» en «Errores relacionados con las definiciones». Por ejemplo, E54 señala como error en definiciones:

En la explicación del método de proporcionalidad (en el ejercicio de las camisas) despeja «X» como si fuese una ecuación. Debería haber sacado la proporción que existe entre las camisas y los metros de tela (cuantas veces es mayor los metros de tela que el número de camisas) y ya dividir entre los metros de tela para sacar la «X».

Después menciona, en «Errores en proposiciones o procedimientos»: «En procedimientos pondría el mismo error que en "Errores en las definiciones"».

La mayoría de los estudiantes que identifican conflictos en procedimientos, hacen referencia a la regla de tres: «Tachar números y despejar la " $x$ " en la regla de tres de forma confusa, escribiendo números pequeños al lado de los grandes que puede dar la sensación de que son potencias» (E52).

Otros estudiantes identifican como errores de procedimiento: «En el método de igualdad de cocientes, situar la incóg- nita "x" en el numerador, siendo más fácil para el alumno colocarlo en el denominador de la fracción izquierda» (E26).

O consideran que las explicaciones que acompañan a los procedimientos son complejas o insuficientes.

Solo un estudiante (E83) hace referencia al error en la proposición: "Para saber si dos magnitudes guardan proporción directa basta comprobar que se cumple que al doble de una le corresponde el doble de la otra, al triple el triple, ...».

Ningún estudiante menciona como imprecisión que la argumentación de la relación de proporcionalidad directa se basa únicamente en la propiedad «al doble de una magnitud corresponde el doble de la otra». En esta categoría, la mayoría de los estudiantes que incluyen algún error lo hacen de forma inapropiada, bien porque indican "explicación confusa», "argumentaciones escasas", bien porque hacen referencia a imprecisiones en la argumentación de transformaciones y no a la relación de proporcionalidad.

Solo seis estudiantes hacen alguna referencia a la falta de argumentación de las operaciones. Por ejemplo, E67 apunta que:

El único inconveniente que le veo a este vídeo es que a la hora de simplificar no argumenta este proceso, es cierto que el vídeo no va sobre simplificación pero en la resolución de problemas puede hacer que los alumnos se pierdan.

Con relación a otros errores, los futuros maestros señalan fundamentalmente: 
- Errores o imprecisiones en la expresión (oral) o lenguaje empleado.

- Presentación no adecuada para alumnos de educación primaria.

- Dificultades para entender las diferencias entre los métodos de resolución del problema.

- Argumentaciones insuficientes para las transformaciones aritméticas.

Respecto al «truco» que presenta el autor del vídeo, E68 incluye como error lo siguiente:

Truco: «Para saber si dos magnitudes guardan proporcionalidad directa, basta comprobar que se cumpla que al doble de una le corresponda el doble de la otra». Aunque es una forma rápida de ver si hay proporcionalidad directa o no, parece impreciso, al hacer solo mención al doble. Habría que mencionar a «mitad, mitad» a «suma de, suma de» a «diferencia de, diferencia de», es decir, aplicado a las cuatro operaciones.

En la Tabla 5 incluimos las frecuencias de errores detectados por los futuros maestros. Distinguimos aquellas respuestas que mencionan de forma algo pertinente un error en la categoría donde se incluye, de aquellas que no lo son porque no se incluye en la categoría indicada, porque la descripción del error no es concluyente 0 es subjetiva, o porque lo que incluyen no se puede considerar error 0 imprecisión. En general, los porcentajes de estudiantes que han reconocido errores e imprecisiones han sido bajos. El más alto ha sido el referido a la justificación de la relación de proporcionalidad, mencionado por 22 estudiantes $(23.7 \%)$.

TABLA 5. Errores identificados por los estudiantes.

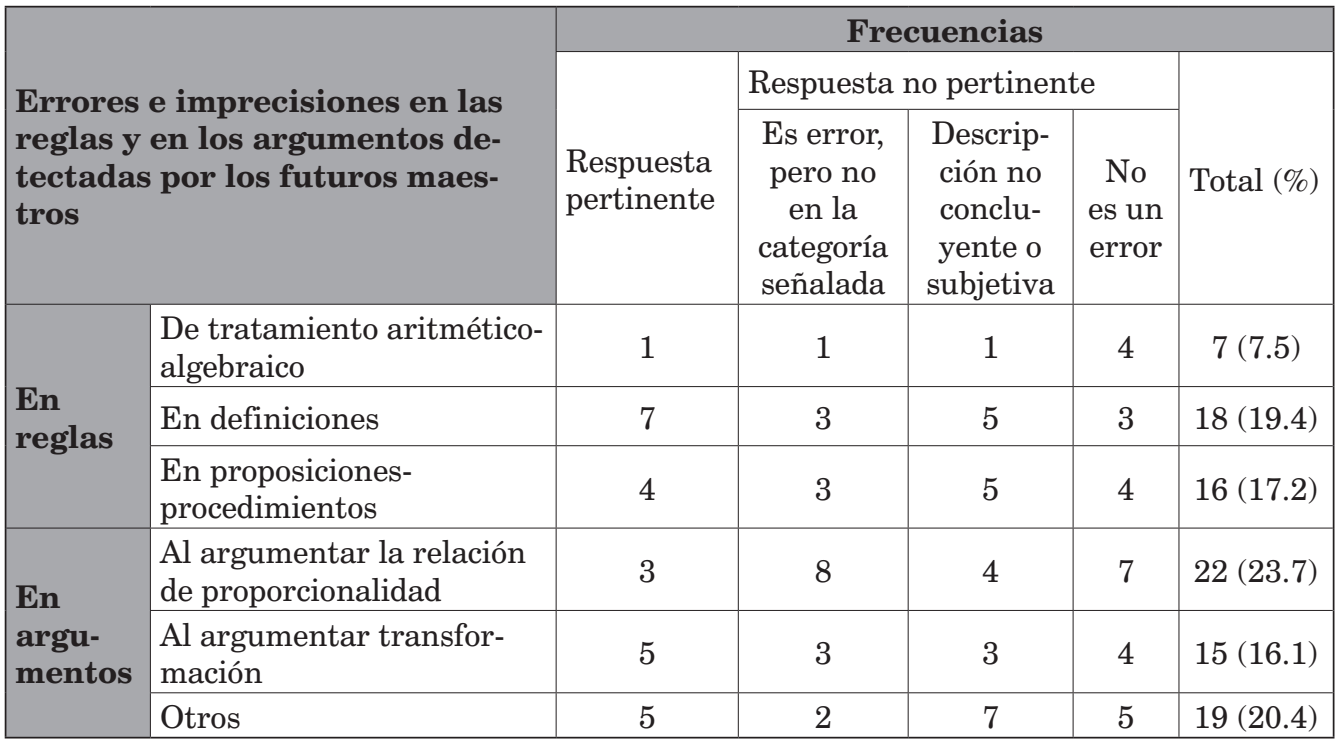

Fuente: Elaboración propia. 
En el apartado d) de la consigna se pide que identifiquen en el vídeo si los objetos matemáticos y los significados se presentan de manera relacionada. Salvo dos estudiantes que no respondieron a este apartado, el $73.12 \%$ de los futuros maestros consideran que los objetos matemáticos se encuentran relacionados de forma pertinente y que, por tanto, el grado de idoneidad en este aspecto es alta (Tabla 6). Por otro lado, el $63.44 \%$ afirma que siempre están articulados los diversos significados de los objetos implicados.

TABLA 6. Relaciones entre objetos y significados.

\begin{tabular}{|c|c|c|c|}
\hline \multicolumn{2}{|c|}{ Relaciones entre los objetos matemáticos } & Idoneidad & Frecuencia (\%) \\
\hline \multirow{3}{*}{ Relaciones entre objetos } & $\begin{array}{l}\text { Todas las proposiciones } \\
\text { y procedimientos tienen } \\
\text { argumento asociado }\end{array}$ & Alta & $68(73.1)$ \\
\hline & $\begin{array}{l}\text { Algunas proposiciones } \\
\text { y procedimientos tienen } \\
\text { argumento asociado }\end{array}$ & Media & $23(24.7)$ \\
\hline & $\begin{array}{l}\text { Ninguna de las propo- } \\
\text { siciones y procedimien- } \\
\text { tos tienen argumento } \\
\text { asociado }\end{array}$ & Baja & $0(0.0)$ \\
\hline \multirow{3}{*}{$\begin{array}{l}\text { Se identifican y articulan } \\
\text { los significados de los obje- } \\
\text { tos que intervienen }\end{array}$} & Siempre & Alta & $59(63.4)$ \\
\hline & A veces & Media & $30(32.3)$ \\
\hline & Nunca & Baja & $2(2.2)$ \\
\hline
\end{tabular}

Fuente: Elaboración propia.

En función de los resultados obtenidos en los análisis previos (variedad y representatividad de las situaciones-problemas propuestas, riqueza de sistemas de representación, claridad y corrección de reglas y argumentos, conexión entre objetos y significados) los futuros maestros debían valorar cuantitativamente el grado de idoneidad epistémica del vídeo. Debían asignar la puntuación $0,1,2$, si consideraban la idoneidad como, baja, media 0 alta, respectivamente.

TABLA 7. Frecuencias (\%) de valoración de la idoneidad epistémica según componentes.

\begin{tabular}{|l|c|c|c|}
\hline \multirow{2}{*}{ Componentes } & \multicolumn{3}{|c|}{ Valoración del vídeo } \\
\cline { 2 - 4 } & $\mathbf{0}$ & $\mathbf{1}$ & $\mathbf{2}$ \\
\hline Situaciones-problema & $1(1.1)$ & $40(43.0)$ & $52(52.9)$ \\
\hline Lenguajes & $3(3.2)$ & $37(39.8)$ & $53(57.0)$ \\
\hline Reglas & $3(3.2)$ & $33(35.5)$ & $57(61.3)$ \\
\hline Argumentos & $1(1.1)$ & $42(45.2)$ & $50(53.8)$ \\
\hline Relaciones entre objetos & $4(4.3)$ & $22(23.7)$ & $67(72.0)$ \\
\hline Articulación de significados & $2(2.2)$ & $37(39.8)$ & $54(58.1)$ \\
\hline
\end{tabular}

Fuente: Elaboración propia. 
Se observa en la Tabla 7 que más de la mitad de los estudiantes asignaron el grado máximo de pertinencia en cada uno de los componentes de la idoneidad epistémica. El aspecto mejor valorado es la relación entre los objetos (el $72.0 \%$ indicaron aquí idoneidad alta) seguido de la corrección de las reglas (61.3\%).

Observemos que la puntuación máxima que se puede asignar al vídeo es de 12 puntos. La puntuación mínima asignada es de 4 puntos (un estudiante) y la media se sitúa en 9.5 puntos. La puntuación más frecuente (en el $29 \%$ de los casos) es de 10 puntos. Además, 9 estudiantes $(10 \%)$ asignaron la máxima puntuación al vídeo.

Se pedía a los futuros maestros que justificaran los motivos que les habían llevado a asignar sus valoraciones de la idoneidad epistémica. El análisis de sus respuestas nos ha permitido encontrar las categorías que mostramos en las Tablas 8 y 9, en las que distinguimos argumentos para una valoración positiva y otros para una valoración negativa.

TABLA 8. Argumentos para dar una valoración positiva (n=93).

\begin{tabular}{|c|c|}
\hline Indicador & Frecuencia $(\%)$ \\
\hline Presentación atractiva & $8(8.6)$ \\
\hline Lenguaje adecuado & $43(46.2)$ \\
\hline Lenguaje variado & $21(22.6)$ \\
\hline $\begin{array}{l}\text { Riqueza de ejemplos (muestra representativa y articulada } \\
\text { de situaciones problema) }\end{array}$ & $26(28.0)$ \\
\hline Situaciones-contexto cotidianas/conexión con vida real & $27(29.0)$ \\
\hline Argumentos adecuados/explicaciones claras & $38(40.9)$ \\
\hline Argumentos suficientes & $17(18.3)$ \\
\hline Procedimientos/métodos diversos y adecuados & $23(24.7)$ \\
\hline Definiciones coherentes/adecuadas & $19(20.4)$ \\
\hline Significados articulados & $17(18.3)$ \\
\hline $\begin{array}{l}\text { Favorece razonamientos o que construyan, perfeccionen y } \\
\text { usen sus propias representaciones para organizar, regis- } \\
\text { trar y comunicar ideas }\end{array}$ & $14(15.1)$ \\
\hline $\begin{array}{l}\text { Relaciones entre objetos (proposiciones con argumento } \\
\text { asociado; métodos relacionados) apropiadas }\end{array}$ & $15(16.1)$ \\
\hline Ningún error en reglas ni argumentos & $7(7.5)$ \\
\hline
\end{tabular}

Fuente: Elaboración propia. 
Los futuros maestros valoran de forma positiva el lenguaje empleado y que los argumentos sean adecuados o las explicaciones claras. Por ejemplo, E14 valora con 2 puntos la componente lenguajes indicando:

El lenguaje utilizado es variado: natural (oral y escrito), simbólico (numérico y algebraico), tabular (a través de tablas), animación (un niño pensando y presenta una pregunta, gif con movimiento en el resultado del problema) y con flechas estableciendo relaciones de proporcionalidad. [...] Además, el nivel del lenguaje es claro y sencillo, adecuado al nivel de Educación Primaria al que va dirigido.

E22 asigna 2 puntos a la componente argumentos:
No he considerado ningún error reseñable referido a los argumentos y a los procedimientos utilizados en el vídeo. En todo momento las operaciones aritméticas se acompañan de una explicación y justificación. Tanto argumentos como procedimientos están claros.

Sin embargo, estos componentes son también los utilizados con mayor frecuencia para dar una valoración negativa, aludiendo tanto a errores de expresión y lenguaje como a argumentos poco claros 0 confusos (Tabla 9). E38 señala: «Algunas veces utiliza un lenguaje un poco técnico para un niño de primaria, los argumentos los veo bien, pero podrían ser un poco más claros en algunos de los 4 métodos que explica de la proporcionalidad directa».

\section{TABLA 9. Argumentos para dar una valoración negativa $(n=93)$.}

\begin{tabular}{|l|c|}
\hline Indicador & Frecuencia (\%) \\
\hline Situaciones-problema no adecuadas a primaria & $3(3.2)$ \\
\hline Errores de expresión/lenguaje & $11(11.8)$ \\
\hline Lenguaje no adaptado al nivel de primaria & $11(11.8)$ \\
\hline $\begin{array}{l}\text { Representaciones pobres (poco conectadas, ausencia de lenguaje } \\
\text { gráfico o poco visual) }\end{array}$ & $3(3.2)$ \\
\hline Dificultad para distinguir métodos & $4(4.3)$ \\
\hline Argumentos excesivos & $3(3.2)$ \\
\hline Argumentos no claros, confusos o rápidos & $19(20.4)$ \\
\hline Proposiciones sin argumento asociado & $1(1.1)$ \\
\hline Definiciones incorrectas o insuficientes & $2(2.2)$ \\
\hline Definiciones no adecuadas al nivel de primaria & $4(4.3)$ \\
\hline Procedimientos no adecuados al nivel de primaria & $3(3.2)$ \\
\hline Procedimientos no claros o rápidos & $4(4.3)$ \\
\hline
\end{tabular}


La cuestión de la idoneidad de los vídeos educativos de matemáticas: una experiencia de ...

\begin{tabular}{|l|c|}
\hline Errores en procedimientos (simplificación en regla de 3) & $5(5.4)$ \\
\hline Argumentos no adaptados al nivel de primaria & $4(4.3)$ \\
\hline Significados no articulados & $12(12.9)$ \\
\hline $\begin{array}{l}\text { Faltan situaciones de generación de significados o representaciones } \\
\text { propias }\end{array}$ & $3(3.2)$ \\
\hline $\begin{array}{l}\text { El autor incluye ideas fuera de contexto o que pueden confundir (por } \\
\text { ejemplo, proporcionalidad inversa/compuesta) }\end{array}$ & $3(3.2)$ \\
\hline Situaciones poco contextualizadas o solo en contexto matemático & $4(4.3)$ \\
\hline
\end{tabular}

Fuente: Elaboración propia.

Todo el análisis debe llevar a los futu- de los estudiantes en «sí», «sí, pero...», ros maestros a decidir si les parece adecua- cuando presentan algún tipo de objeción, y do el vídeo argumentando su decisión. En «no». La Tabla 10 incluye las frecuencias y este caso, hemos clasificado las respuestas porcentajes de cada una de estas opciones.

TABLA 10. Adecuación del vídeo en la reflexión final (n=93).

\begin{tabular}{|l|c|}
\hline ¿Te parece adecuado este vídeo? & Frecuencia (\%) \\
\hline Sí & $48(51.61)$ \\
\hline Sí, pero... & $38(40.86)$ \\
\hline No & $7(7.53)$ \\
\hline
\end{tabular}

Fuente: Elaboración propia.

El $51.61 \%$ de los futuros maestros con- do de adecuación de las explicaciones. La sideran adecuado el vídeo, siendo lo más Tabla 11 resume los distintos argumentos valorado por estos la presencia de varias empleados por los estudiantes para valorar formas de resolver los problemas y el gra- como adecuado el vídeo.

TABLA 11. Argumentos cuando consideran totalmente adecuado el vídeo $(\mathrm{n}=48)$.

\begin{tabular}{|l|c|}
\hline Indicador & Frecuencia (\%) \\
\hline Varias formas de resolver & $46(95.8)$ \\
\hline Riqueza de ejemplos & $22(45.8)$ \\
\hline Explicación adecuada y detallada & $42(87.5)$ \\
\hline $\begin{array}{l}\text { Simplifica las definiciones/presenta la información más relevante y } \\
\text { representativa }\end{array}$ & $13(27.1)$ \\
\hline
\end{tabular}




\begin{tabular}{|l|c|}
\hline Representación/lenguaje adecuado & $26(54.2)$ \\
\hline Utilidad/motivador & $7(14.6)$ \\
\hline Incluye trucos/consejos & $12(25.0)$ \\
\hline Contexto cercano & $10(10.8)$ \\
\hline Lleva a reflexionar sobre el método más adecuado & $6(12.5)$ \\
\hline No hay errores & $7(14.6)$ \\
\hline Duración adecuada & $2(4.2)$ \\
\hline Presentación atractiva, tono informal & $5(10.4)$ \\
\hline
\end{tabular}

Fuente: Elaboración propia.

El $40.86 \%$ de los futuros maestros métodos empleados como poco adecuados no consideran del todo adecuado el vídeo para primaria, o que puede ser pesado (Tabla 10). Señalan como inconvenientes para los alumnos. la extensión, el lenguaje 0 algunos de los

TABLA 12. Argumentos que indican inconvenientes («sí, pero...») (n=38).

\begin{tabular}{|l|c|}
\hline \multicolumn{1}{|c|}{ Indicador } & Frecuencia (\%) \\
\hline Lenguaje no adecuado a primaria & $11(28.9)$ \\
\hline No detallan procedimientos aritméticos & $4(10.5)$ \\
\hline Duración excesiva & $6(15.8)$ \\
\hline Llega a aburrir o desconcentrar, falta animación o recursos visuales & $19(50)$ \\
\hline Provoca confusión en los alumnos & $6(15.8)$ \\
\hline Falta variedad de contextos & $3(7.9)$ \\
\hline Explicaciones no acertadas & $5(13.2)$ \\
\hline Largo y denso para primaria & $11(28.9)$ \\
\hline No todas las situaciones/conceptos/métodos son adecuados a primaria & $12(31.6)$ \\
\hline No toma en cuenta conocimientos previos o dificultades de aprendizaje & $4(10.5)$ \\
\hline Presencia de errores & $7(18.4)$ \\
\hline
\end{tabular}

Fuente: Elaboración propia. 
Finalmente, solo el $7.5 \%$ de los estudiantes no considera adecuado el vídeo. Aducen que las definiciones son poco 0 nada precisas y las explicaciones son inadecuadas 0 confusas para primaria.

El análisis de las respuestas de los estudiantes nos ha permitido detectar algunos conflictos con relación a la identificación de componentes y descriptores en el análisis de la idoneidad epistémica, fundamentalmente en lo que a reglas y articulación de significados se refiere. Por ejemplo, E32 identifica reglas con formas de resolver un problema y afirma: «Las reglas las he valorado con un 2 porque sí es verdad que muestra gran variedad de formas de resolver el problema».

Similarmente, E56 señala que:

En cuanto a las reglas he establecido una puntuación de 2 (alta) de evaluación de idoneidad, puesto que estoy de acuerdo en la forma en que realiza las operaciones en cada una de las resoluciones del problema y porque no creo que cometa ningún error al llevarlas a cabo.

Algunos estudiantes consideran que los significados están articulados cuando se utilizan varios métodos o se justifica lo que se está haciendo en el vídeo. Por ejemplo, E41 indica: «La articulación de significados considero que tiene un nivel alto, debido que utiliza varios métodos para la resolución de los problemas».

E30 incluye: «Para la articulación de significados la idoneidad es alta, ya que explica en todo momento lo que significa cada cosa que está realizando».

\section{Síntesis, implicaciones y limi- taciones}

El objetivo de este trabajo ha sido el diseño, implementación y evaluación de una acción formativa con futuros maestros centrada en el desarrollo de conocimientos y competencias para el análisis de la idoneidad epistémica de vídeos educativos sobre proporcionalidad. Se ha comenzado con plantear el interés del tema, dada la abundancia y disponibilidad de vídeos que se ofrecen como recurso para favorecer la enseñanza de las matemáticas. No obstante, teniendo en cuenta la desigual calidad y diversidad de significados de los vídeos educativos (Beltrán-Pellicer, Giacomone y Burgos, 2018) se justifica la necesidad de capacitar a los profesores para la valoración y uso pertinente de estos recursos.

La acción formativa se ha orientado a proporcionar a los futuros maestros una herramienta teórica para el análisis de la idoneidad epistémica, esto es, del contenido matemático presentado en un vídeo sobre proporcionalidad. El análisis a priori del vídeo hecho por los investigadores reveló errores e imprecisiones en las definiciones, proposiciones y procedimientos, así como carencia o inexactitud en los argumentos ofrecidos para justificar los procedimientos y proposiciones. También la presentación y tratamiento de una variedad de situaciones-problemas y la articulación de significados de la proporcionalidad tiene carencias importantes, por lo que el nivel de idoneidad epistémica se valora como media en la escala ordinal baja, media, alta. Sin embargo, la mayoría de los futuros docentes, tras el proceso formativo aplicado, valoraron el 
grado de idoneidad del vídeo como alto en casi todos los componentes.

El instrumento de evaluación usado, basado en los componentes e indicadores de la idoneidad epistémica, tiene en cuenta la variedad de situaciones-problemas propuestos, la presencia de distintos registros de representación, la claridad y corrección de las definiciones, las proposiciones y procedimientos, así como la justificación de los procedimientos y proposiciones con argumentos pertinentes y adaptados al nivel educativo correspondiente. El no haber reconocido la ausencia de algunos indicadores importantes ha llevado a un porcentaje elevado de estudiantes $(51.6 \%)$ a considerar el vídeo como un recurso didáctico adecuado, sin identificar sus carencias.

Por otro lado, hemos constatado cierta variabilidad en la asignación de las puntuaciones por los participantes, encontrando valoraciones con la máxima puntuación en las que el estudiante señala más inconvenientes que en otras con menos valoración. Si bien estas fluctuaciones mantienen coherente la valoración global, nos indica que quizá sea necesario concretar más los criterios, con el objetivo de perseguir una mayor uniformidad inter-participantes. No obstante, hemos de tener en cuenta que se trata de una valoración cualitativa de la idoneidad y que el número final, dentro de unos márgenes, es solo el resultado sintético de un proceso más complejo, donde se han identificado los diferentes componentes de la idoneidad.

Estos resultados sugieren la necesidad de profundizar en el desarrollo del cono- cimiento especializado del contenido de los futuros profesores, en este caso, sobre proporcionalidad, ampliando el tiempo de instrucción asignado, analizando una mayor variedad de vídeos educativos e incrementando la fase de discusión colectiva de los resultados de los análisis.

Este tipo de acciones formativas, centradas en el contenido de la disciplina, pero con una clara orientación hacia el conocimiento y las competencias didácticas, se alinean con trabajos de otros autores (Davis, 2015), donde se señala la repercusión que tiene su estudio del concepto tanto a la hora de revelar la complejidad de las ideas matemáticas subyacentes como del desarrollo de las matemáticas necesarias para la enseñanza.

Por otra parte, la herramienta teórica de la idoneidad didáctica incluye, además de la faceta epistémica, las facetas cognitiva, afectiva, interaccional, mediacional y ecológica, las cuales no han sido abordadas en esta investigación. Aunque no es pertinente aplicar algunas de estas facetas en el caso del uso de recursos didácticos, en particular la faceta de los aprendizajes logrados, los demás aspectos podrían ser objeto de análisis y reflexión por parte de los profesores que usen estos recursos.

\section{Notas}

1 El vídeo del canal Clasemáticas puede verse en https://www.youtube.com/watch?v=01Mu- Ikgv-0 ${ }^{2}$ Los vídeos que se propusieron pueden verse en https://www.youtube.com/watch?v=0Z5DejetHR8 https://www.youtube.com/watch?v=v8KN44iNPIs https://www.youtube.com/watch?v=1uAblb-McLo 


\section{Referencias bibliográficas}

Aroza, C. J., Godino, J. D. y Beltrán-Pellicer, P. (2016). Iniciación a la innovación e investigación educativa mediante el análisis de la idoneidad didáctica de una experiencia de enseñanza sobre proporcionalidad. Avances en Investigación e Innovación - Revista de Educación Secundaria, 6 (1), 1-29.

Azer, S. A., AlGrain, H. A., AlKhelaif, R. A. y AlEshaiwi, S. M. (2013). Evaluation of the Educational Value of YouTube Videos about Physical Examination of the Cardiovascular and Respiratory Systems. Journal of Medical Internet Research, 15 (11), e241. doi: https:// doi.org/10.2196/jmir.2728

Bartell, T. G., Webel, C., Bowen, B. y Dyson, N. (2013). Prospective teacher learning: recognizing evidence of conceptual understanding. Journal of $M a$ thematics Teacher Education, 16 (1), 57-79.

Beltrán-Pellicer, P., Giacomone, B. y Burgos, M. (2018). Online educational videos according to specific didactics: the case of mathematics. $\mathrm{Cul}$ tura y Educación, 30 (4), 633-662.

Beltrán-Pellicer, P., Godino, J. D. y Giacomone, B. (2018). Elaboración de indicadores específicos de idoneidad didáctica en probabilidad: aplicación para la reflexión sobre la práctica docente. Bolema, 32 (61), 526-548.

Ben-Chaim, D., Keret, Y. e Ilany, B. (2012). Ratio and proportion: Research and teaching in mathematics teachers' education. Rotterdam: Sense Publisher.

Bergmann, J. y Sams, A. (2012). Flip your classroom. Eugene, Oregon: International Society for Technology in Education.

Berk, D., Taber, S. B., Gorowara, C. C. y Petzl, C. (2009). Developing prospective elementary teachers' flexibility in the domain of proportional reasoning. Mathematical Thinking and Learning, 11 (3), 113-135.

Borba, M. C., Askar, P., Engelbrecht, J., Gadanidis, G., Llinares, S. y Aguilar, M. S. (2016). Blended learning, e-learning and mobile learning in mathematics education. ZDM. Mathematics Education, 48 (5), 589-610.
Breda, A., Pino-Fan, L. y Font, V. (2017). Meta didactic-mathematical knowledge of teachers: criteria for the reflections and assessment on teaching practice. Eurasia Journal of Mathematics, Science \& Technology Education, 13 (6), 1893-1918.

Chapman, 0. (2014). Overall commentary: understanding and changing mathematics teachers. En J.-J. Lo, K. R. Leatham y L. R. Van Zoest (Eds.), Research Trends in Mathematics Teacher Education (pp. 295-309). Dordrecht: Springer International Publishing.

Clasemáticas Canal (8 de noviembre de 2012). (Proporcionalidad) - Proporción Directa (1002) [Archivo de vídeo]. Recuperado de https://www. youtube.com/watch? $\mathrm{v}=01 \mathrm{Mu}-\mathrm{lkgv}-0$ (Consultado el 06-04-2019).

Dabbagh, B. y Kitsantas, A. (2012). Personal Learning Environments, social media, and self-regulated learning: A natural formula for connecting formal and informal learning. The Internet and Higher Education, 12 (1), 3-8.

Daniel Carreon (27 de junio de 2017). REPARTO PROPORCIONAL - Super fácil [Archivo de vídeo]. Recuperado de https://www.youtube.com/ watch?v=1uAbIb-McLo (Consultado el 06-042019).

Davis, B. (2015). Las matemáticas que los profesores de educación secundaria conocen (o necesitarían conocer). revista española de pedagogía, 73 (261), 321-342.

Duffy, P. (2008). Engaging the YouTube Googleeyed generation: Strategies for using Web 2.0 in teaching and learning. Electronic Journal of E-learning, 6 (2), 119-130.

English, L. D. (2008). Setting an agenda for international research in mathematics education. En L. D. English y D. Kirshner (Eds.), Handbook of international research in mathematics education (pp. 3-19). Nueva York y Londres: Taylor and Francis (Routledge).

Godino, J. D. (2013). Indicadores de idoneidad didáctica de procesos de enseñanza y aprendizaje de las matemáticas. Cuadernos de Investigación y Formación en Educación Matemática, 11, 111-132. 
Godino, J. D., Batanero, C. y Font, V. (2007). The onto-semiotic approach to research in mathematics education. ZDM. Mathematics Education, 39 (1-2), 127-135.

Godino, J. D., Font, V., Wilhelmi, M. R. y Lurduy, 0. (2011). Why is the learning of elementary arithmetic concepts difficult? Semiotic tools for understanding the nature of mathematical objects. Educational Studies in Mathematics, 77 (2), 247-265.

Godino, J. D., Giacomone, B., Batanero, C. y Font, V. (2017). Enfoque ontosemiótico de los conocimientos y competencias del profesor de matemáticas. Bolema, 31 (57), 90-113.

Hilton, A. y Hilton, G. (2018). Primary school teachers implementing structured mathematics interventions to promote their mathematics knowledge for teaching proportional reasoning. Journal of Mathematics Teacher Education, 22 (6), 545-574. doi: https://doi.org/10.1007/ s10857-018-9405-7

Mason, J. (2016). Perception, interpretation and decision making: understanding gaps between competence and performance-a commentary. ZDM. Mathematics Education, 48 (1-2), 219226.

Pino-Fan, L. y Godino, J. D. (2015). Perspectiva ampliada del conocimiento didáctico-matemático del profesor. Paradigma, 36 (1), 87-109.

Ponte, J. P. y Chapman, O. (2016). Prospective mathematics teachers' learning and knowledge for teaching. En L. D. English y D. Kirshner (Eds.), Handbook of international research in mathematics education (pp. 275-296). Nueva York: Routledge.

Portugal, K. O., Arruda, S. D. M. y Passos, M. M. (2018). Free-choice teaching: how YouTube presents a new kind of teacher. Revista Electrónica de Enseñanza de las Ciencias, 17 (1), 183-199.

Posadas, P. y Godino, J. D. (2017). Reflexión sobre la práctica docente como estrategia formativa para desarrollar el conocimiento didáctico-matemático. Didacticae, 1, 77-96. doi: https://doi. org/10.1344/did.2017.1.77-96

Ramírez, A. (2010). Youtube y el desarrollo de la competencia matemática. Resultados de una investigación cuasi-experimental. Contextos Educativos, 13, 123-138.
Ruiz-Reyes, K., Contreras, J. M., Arteaga, P. y Oviedo, K. (2017). Análisis semiótico de videos tutoriales para la enseñanza de la probabilidad en educación primaria. En J. M. Contreras, P. Arteaga, G. R. Cañadas, M. M. Gea, B. Giacomone y M. M. López-Martín (Eds.), Actas del Segundo Congreso International Virtual sobre el Enfoque Ontosemiótico del Conocimiento y la Instrucción Matemáticos. Recuperado de http:// hdl.handle.net/10481/45382 (Consultado el 1907-2019).

Sadler, D. R. (2013). Making competent judgments of competence. En S. Blömeke, O. Zlatkin-Troitschanskaia, C. Kuhn y J. Fege (Eds.), Modeling and measuring competencies in higher education: Tasks and challenges (pp. 1327). Rotterdam: Sense Publishing.

Santos, J. A. (2018). Valoración de vídeo tutoriales de matemáticas disponibles en Internet. Nuevos instrumentos para el análisis de los procesos educativos (Tesis doctoral). Centro de Investigación y de Estudios Avanzados del Instituto Politécnico Nacional, Ciudad de México, México.

Smatematico (7 de abril de 2013). Problema de reparto proporcional [Archivo de vídeo]. Recuperado de https://www.youtube.com/watch?v=0Z5DejetHR8 (Consultado el 06-042019).

Turney, C. S. M., Robinson, D., Lee, M. y Soutar, A. (2009). Using technology to direct learning in higher education. The way forward? Active Learning in Higher Education, 10 (1), 71-83.

Tuto mate (10 de mayo de 2016). Problemas de repartos directamente proporcionales [Archivo de vídeo]. Recuperado de https://www.youtube. com/watch?v=v8KN44iNPls (Consultado el 06-04-2019).

\section{Biografía de los autores}

María Burgos es Profesora Contratada Doctora en el Departamento de Didáctica de la Matemática de la Universidad de Granada. Doctora en Matemáticas por la Universidad de Almería y Máster en Didáctica de la Matemática por la Univer- 
sidad de Granada. Es miembro del grupo FQM-126 Teoría de la Educación Matemática y Educación Estadística.

(iD https://orcid.org/0000-0002-4598-7684

Pablo Beltrán-Pellicer es Profesor Asociado en el área de Didáctica de las Matemáticas de la Universidad de Zaragoza y Profesor de Educación Secundaria Obligatoria y Bachillerato en el Colegio Público Integrado Val de la Atalaya (María de Huerva, Zaragoza). Doctor en Didáctica de las Matemáticas y miembro del grupo S36_17D Investigación en Educación Matemática.

(iD https://orcid.org/0000-0002-1275-9976

Juan D. Godino es Catedrático jubilado de Didáctica de la Matemática, Colaborador Extraordinario del Departamento de Didáctica de la Matemática y Profesor del programa de Doctorado en Educación de la Universidad de Granada. Miembro del grupo FQM-126 Teoría de la Educación Matemática y Educación Estadística. Desde 1993 viene desarrollando el Enfoque Ontosemiótico del conocimiento y la instrucción matemáticos, un marco teórico específico para la educación matemática.

(iD http://orcid.org/0000-0001-8409-0258 


\section{revista española de pedagogía} año 78, n² 275, enero-abril 2020

Spanish Journal of Pedagogy

year 78, n. 275, January-April 2020

\section{Sumario*}

\section{Table of Contents **}

\section{Cuestiones de enseñanza de las matemáticas}

Wendolyn Elizabeth Aguilar-Salinas, Maximiliano de las Fuentes-Lara, Araceli Celina Justo-López y Ana Dolores Martínez-Molina

Instrumento de medición para diagnosticar las habilidades algebraicas de los estudiantes en el Curso de Cálculo Diferencial en ingeniería

A measurement instrument for establishing the algebraic skills of engineering students on a Differential Calculus Course in engineering

Maria Burgos, Pablo Beltrán-Pellicer y Juan D. Godino La cuestión de la idoneidad de los vídeos educativos de matemáticas: una experiencia de análisis con futuros maestros de educación primaria

The issue of didactical suitability in mathematics educational videos: experience of analysis with prospective primary school teachers

\section{La formación online en el mundo de la educación: experiencias de los Estados Unidos}

Gerald LeTendre y Tiffany Squires

Integración de programas de máster online y presenciales en educación

Integrating online and residential master's programs in education

\section{Laurence B. Boggess}

Innovación en la capacitación docente online: un modelo organizacional para brindar apoyo a largo plazo a la docencia online Innovations in online faculty development: an organizational model for long-term support of online faculty

Brian Redmond

Liderazgo de equipo compartido de un programa online

27 Shared team leadership for an online program

\footnotetext{
* Todos los artículos están también publicados en inglés en la página web de la revista: https://revistadepedagogia.org.

** All the articles are also published in English on the web page of the journal: https://revistadepedagogia.org.
} 


\section{Estudios y Notas}

\section{Paolo Scotton}

Pensar en común, vivir en plenitud. La experiencia de

la filosofía con los niños y niñas

Thinking together, living fully. Experiencing philosophy with

children

103

\section{Manuel Delgado-Garcia, Sara Conde Vélez y Ángel}

Boza Carreño

Perfiles y funciones del tutor universitario y sus

efectos sobre las necesidades tutoriales del alumnado

Profiles and functions of university tutors and their effects on

students' tutorial needs

119

\section{Reseñas bibliográficas}

Luri, G. (2019). La imaginación conservadora: una defensa apasionada de las ideas que han hecho del mundo un lugar mejor (Enrique Alonso Sainz).

Quigley, C. F. y Herro, D. (2019). An educator's guide to STEAM. Engaging students using real-world problems (Juan Luis Fuentes).Prince, T. (2019). Ejercicios de mindfulness en el aula. 100 ideas prácticas (José V. Merino Fernández).

\section{Informaciones}

Francisco Altarejos: la filosofía de la educación hecha vida (Concepción Naval); World Educational Research Association Focal Meeting 2020: «Networking Education: Diverse Realities, Common Horizons»);

Conclusiones del IX Congreso Internacional de Filosofía de la Educación; Ebook 2019: José Antonio Ibáñez-Martín (Ed.) (2019). La misión de las revistas de investigación en el mundo educativo. The mission of research journals in the world of education.

\section{Instrucciones para los autores}

Instructions for authors

\section{Solicitud de originales}

Call for papers

169

XV Congreso Internacional de Teoría de la Educación

XV International Congress on Theory of Education

ISSN: 0034-9461 (Impreso), 2174-0909 (Online)

https://revistadepedagogia.org/

Depósito legal: M. 6.020 - 1958

INDUSTRIA GRÁFICA ANZOS, S.L. Fuenlabrada - Madrid 


\section{The issue of didactical suitability in mathematics educational videos: experience of analysis with prospective primary school teachers}

\section{La cuestión de la idoneidad de los vídeos educativos de matemáticas: una experiencia de análisis con futuros maestros de educación primaria}

María BURGOS, PhD. Associate Lecturer. Universidad de Granada (mariaburgos@ugr.es).

Pablo BELTRÁN.PELLICER, PhD. Associate Lecturer. Universidad de Zaragoza (pbeltran@unizar.es). Juan D. GODINO, PhD. Professor. Universidad de Granada (jgodino@ugr.es).

\section{Abstract:}

The number of educational videos available on the internet on the most varied topics is rapidly increasing. These include mathematics videos that cover virtually any type of curriculum content. However, their quality as a learning resource varies greatly. As a result, it is necessary to provide teachers with tools to enable them to analyse the appropriateness of using educational videos, considering the various aspects involved. This paper describes the design, implementation and results of an educational intervention with 93 prospective primary school teachers, focussed on developing their ability to analyse the educational suitability of videos about proportionality. Preliminary analysis of the video revealed significant errors and inaccuracies in the definitions, propositions, and procedures, as well as shortcomings and inaccuracies in the arguments, and so its level of epistemic suitability is rated as medium. However, the majority of the prospective teachers rated its degree of suitability as high in almost all components.

This work was carried out in the framework of the EDU2016-74848-P (Spanish state research agency, ERDF) research project and FQM-126 (Regional Government of Andalucia) and S36_17D (Regional Government of Aragón and European Social Fund) groups, Spain.

Revision accepted: 2019-11-25.

This is the English version of an article originally printed in Spanish in issue 275 of the revista española de pedagogía. For this reason, the abbreviation EV has been added to the page numbers. Please, cite this article as follows: Burgos, M., Beltrán-Pellicer, P., \& Godino, J. D. (2020). La cuestión de la idoneidad de los vídeos educativos de matemáticas: una experiencia de análisis con futuros maestros de educación primaria | The issue of didactical suitability in mathematics educational videos: experience of analysis with prospective primary school teachers. Revista Española de Pedagogía, 78 (275), 27-49. doi: https://doi.org/10.22550/REP78-1-2020-07 
Students regard studying didactic suitability and implementing it through components and indicators as positive, believing that this activity facilitates professional reflection. However, mastering this tool requires analysing a greater number and variety of videos and further collective discussion of the results of the analyses performed by the students.

Keywords: teachers' education, onto-semiotic approach, didactical suitability, educational videos, proportionality.

\section{Resumen:}

La cantidad de vídeos educativos disponibles en Internet sobre los más variados temas está aumentando a un ritmo acelerado. Así, nos encontramos vídeos de matemáticas que cubren prácticamente cualquier tópico curricular, aunque su calidad como recurso didáctico es muy desigual. En consecuencia, es necesario proporcionar a los profesores herramientas que les permitan analizar la pertinencia del uso de estos vídeos, teniendo en cuenta los diversos aspectos implicados. En este trabajo se describe el diseño, imple- mentación y resultados de una acción formativa con 93 futuros maestros de educación primaria, orientada al desarrollo de la competencia de análisis de la idoneidad didáctica de vídeos sobre proporcionalidad. El análisis a priori del vídeo reveló errores e imprecisiones significativas en las definiciones, proposiciones y procedimientos, así como carencias 0 inexactitudes en los argumentos, por lo que el nivel de idoneidad epistémica se valora como media. Sin embargo, la mayoría de los futuros docentes valoraron su grado de idoneidad como alto en casi todos los componentes. Los estudiantes consideran positivo el estudio de la idoneidad didáctica y su implementación a través de componentes e indicadores, considerando que esta actividad facilita la reflexión profesional. No obstante, el dominio de esta herramienta requiere incrementar el número y variedad de vídeos para analizar y mayor discusión colectiva de los resultados de los análisis que realizan los estudiantes.

Descriptores: formación de profesores, enfoque ontosemiótico, idoneidad didáctica, vídeos educativos, proporcionalidad.

\section{Introduction}

The use of educational videos from YouTube and other platforms has increased dramatically in recent years, offering a promising learning resource for students and the general public (Azer, AlGrain, AlKhelaif, \& AlEshaiwi, 2013).

These educational resources and the pedagogical models that use them, such as flipped learning (Bergmann \& Sams, 2012), should be the subject of educational research as it is not clear how possible it is to achieve meaningful learning by watching recorded classes. Indeed, several researchers discuss the role that the use of YouTube and other social media might play in formal education, analysing how online resources are organised and how they can be inserted 
as informal tools in specific educational settings (Borba et al., 2016; Dabbagh \& Kitsantas, 2012; Duffy, 2008; Portugal, Arruda, \& Passos, 2018; Ramírez, 2010). It is widely regarded as necessary for the field of educational studies to investigate the adequacy of online educational resources to ensure that technology is in accordance with learning objectives (Turney, Robinson, Lee, \& Soutar, 2009).

Research in mathematics teaching regarding the use of educational videos emphasises the importance of the teachers themselves evaluating and recommending suitable videos for their pupils (Beltrán-Pellicer, Giacomone, \& Burgos, 2018; Ruiz-Reyes, Contreras, Arteaga, \& Oviedo, 2017; Santos, 2018). This is because some of these videos show formally incorrect procedures, not all of them indicate the educational level for which they are intended, and the meanings they raise might not be relevant to what is being covered in class. Accordingly, there is a clear need to design and implement training processes that make it possible to promote teachers' professional growth and develop their knowledge and competences (Chapman, 2014; English, 2008; Mason, 2016; Ponte \& Chapman, 2016; Sadler, 2013).

In view of this issue, the aim of this research is to design, implement, and evaluate a training activity for future primary school teachers, focussing on developing knowledge and competences, relating to the analysis of the epistemic suitability of educational videos about proportionality that are available on the internet.

The work is based around the following sections. Section 2 outlines the theoretical framework and specific research problem. Section 3 describes the design of the training process implemented. Section 4 includes a preliminary analysis of the video about proportionality, which is used as an instrument for evaluating the competences achieved by the future teachers. Section 5 shows in detail the results of the experiment, analysing qualitatively and quantitatively the reports drawn up individually by the future teachers. The final section includes a summary of the research and discussion of its implications, and limitations.

\section{Theoretical framework and re- search problem}

In the field of research into training mathematics teachers, different theoretical frameworks are used to categorise and promote different types of professional knowledge and competences (Pino-Fan \& Godino, 2015). We consider that the didactic-mathematical knowledge and competences (DMKC) model (Godino, Giacomone, Batanero, \& Font, 2017; Breda, Pino-Fan, \& Font, 2017), developed within the framework of the onto-semiotic approach to mathematical knowledge and instruction (OSA) (Godino, Batanero, \& Font, 2007), provides suitable tools for approaching our research problem. This model emphasises the importance of designing and implementing training resources that promote teachers' competence in 
analysing educational suitability. Didactical suitability is understood as the degree to which an instructional process combines certain characteristics that mean it can be described as appropriate or adequate, the main criterion being the fit between the personal meanings students construct (learning) and the institutional meanings, whether these are intended or actually implemented (teaching), taking into account the influence of the environment (Godino, 2013). This involves coherently and systematically articulating six criteria relating to the facets that affect a training process (Godino et al., 2007): epistemic, ecological, cognitive, emotional, interactive, and those relating to media.

We consider that a mathematics training process is more epistemically suitable the better the institutional meanings implemented (or intended) represent a reference meaning. The reference meaning will relate to the particular educational level and should be drawn up, taking into account the different types of problems and contexts for use of the content taught, as well as the operational and discursive practices required (Godino, 2013). Consequently, it will be necessary to take into account the degree of adequacy of the situations-problems and it will also be necessary to consider the variety and adequacy of the representations, definitions, procedures, and propositions, as well as the arguments supporting them. High suitability from an epistemic perspective requires the situations-problems proposed to involve a variety of representations, to offer students a variety of ways of approaching them, and to require students to interpret, generalise, and justify the solutions. Furthermore, the different partial meaning of the mathematical objects that appear must be connected and defined (Godino, Font, Wilhelmi, \& Lurduy, 2011).

Using didactical suitability enables teachers to reflect systematically on their own practice (Aroza, Godino, \& BeltránPellicer, 2016; Beltrán-Pellicer, Godino, \& Giacomone, 2018; Posadas \& Godino, 2017) and it can also be applied to analyse partial aspects of the instructional processes, such as the use of technological resources. Specifically, Beltrán-Pellicer, Giacomone, and Burgos (2018), using the theoreticalmethodological tools from OSA, analysed the degree of epistemic suitability of a selection of the educational videos viewed most frequently by users of YouTube ${ }^{\mathrm{TM}}$, relating to problems of proportional sharing. On the one hand, they found a wide variety of focuses and methods for solving this type of problem, which can interfere with the teaching and learning process in the classroom if the teacher does not previously select or record these videos. On the other hand, the epistemic suitability of the sample of videos analysed was very diverse, with videos that contained errors and inaccuracies as well as many of them offering an unrepresentative or poorly defined treatment of the mathematical content. Finally, they noted that the videos with the metrics reflecting the greatest popularity were not the most suitable ones. This work is the main antecedent of the present paper.

We consider that it would be desirable for teachers to understand the didactic 
suitability of the tool and acquire the necessary competence to use it to analyse educational resources critically, particularly in the use of videos available online.

Furthermore, various pieces of research show that teachers, both in their initial training and in service, show difficulties with teaching concepts relating to proportionality (Bartell, Webel, Bowen, \& Dyson, 2013; Ben-Chaim, Keret, \& Ilany, 2012; Berk, Taber, Gorowara, \& Poetzl, 2009; Hilton \& Hilton, 2018). Teacher training must take into account the development of mathematics teaching knowledge and competences relating to this topic by designing and implementing specific training interventions. This is why the trainee teachers were asked to analyse a video on proportionality.

\section{Design of the training process}

The training experience was carried out during the 2018-2019 academic year in the framework of the Curriculum Design and Development in Primary Education module with ninety-third year students from the Primary Education degree.

During their degree studies, future teachers receive specific training about epistemic (mathematical content), cognitive (mathematics learning, errors, and difficulties), instructional, and curricular aspects, so that when carrying out their school placements, they should be able to put into practice the knowledge they have acquired to analyse, design, and support teaching-learning according to specific content (in our case, proportionality).
Furthermore, before carrying out this research, and in line with the chosen DMKC model, we carried out training workshops with the group of students focussing on developing the competence of analysing global meaning (based on identifying situations-problems and the operational, discursive, and normative practices involved in solving them), and onto-semiotic analysis of the practices (description of the framework of objects and processes involved in the practices) used in the mathematical activity of solving problems involving proportionality.

The first session comprised a two-hour workshop where the characteristics of the theory of didactical suitability were presented, as well as how the different epistemic, cognitive, affective, interactional, media related, and ecological dimensions of a given study process relate to each other. The aim was to involve future primary school teachers in a reflection on the need to have a system of specific indicators that make it possible to evaluate teaching practice systematically.

In the next session, also lasting two hours, the future teachers worked in teams to analyse the epistemic suitability of online educational videos on proportionality. The initial group work enabled the students to compare, discuss, and improve their proposals for evaluating the epistemic suitability of different educational videos relating to proportionality.

In the third phase, the students individually completed the tasks described in 
the following section as a final evaluation instrument, the results of which are analysed in this work.

\section{Preliminary analysis of the epistemic suitability of the video}

In this section we analyse the epistemic suitability of the video, that is, the mathematical knowledge brought into play in it. This analysis will serve as a benchmark for interpreting the students' answers when they evaluate the suitability of the educational video. The researchers performed the analysis and preliminary evaluation independently. They then compared them to decide on a common evaluation.

The educational video analysed ${ }^{1}$ covers the topic of direct arithmetic proportionality from the perspective of presenting the notions of ratio and proportion (BenChaim, Keret, \& Ilany, 2012). The idea is that the future teachers will watch the video closely and critically assess its degree of suitability, in line with the epistemic suitability components and indicators (Godino et al., 2007; Godino, 2013).
In general, the situations-problems proposed in the video are presented in context and the mathematical ideas are connected. Various ways of approaching the problems are proposed, but we consider that the sample of the problems is not sufficiently representative or defined. The solution methods proposed are only applied to problems with a missing value, in which the condition of regularity is assumed in advance, and problems involving comparing ratios, for example, are not considered. The video features a great variety of linguistic registers and representations: natural registers (oral and written), symbolic (numerical and algebraic), tabular, and graphic ones.

Furthermore, we have identified some errors and inaccuracies in the presentation of rules (definitions and propositions) and arguments:

- Error of expression in the handling of simplifications of fractions. To simplify, the numbers that appear in the numerator and denominator are crossed out, leaving as superscript the factors that remain when canceling terms.

\section{GRAPH 1. Screenshot from minute 7.}

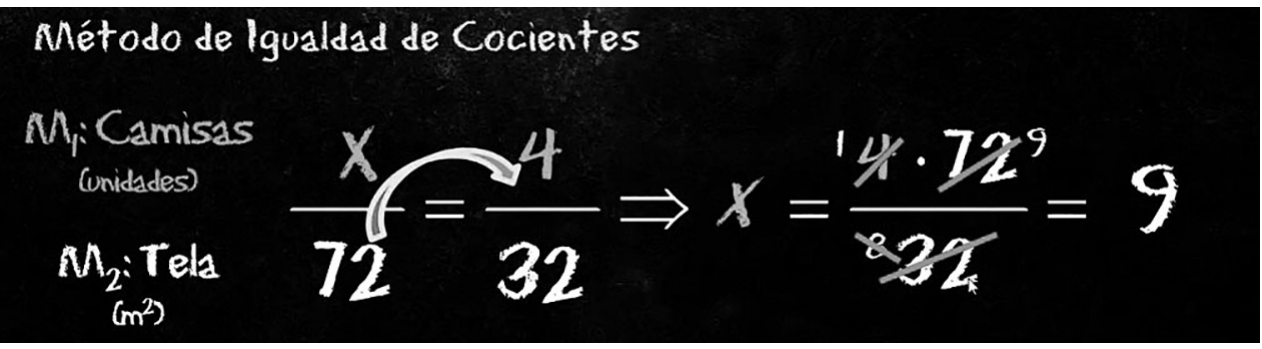

Image text: Quotient Equality Method. $\mathrm{M}_{1}$ : shirts (units) $\mathrm{M}_{2}$ : fabric $\left(\mathrm{m}^{2}\right)$ 
- Error in the definition of ratio and proportion.

(1:00) «Ratio, mathematically speaking, means quotient. The proportion, the ratio, the quotient between these two magnitudes is always the same.»

Here we see incorrect use of the concepts of ratio and proportion. A ratio is not always a quotient and a ratio is not the same as a proportion, a confusion found with some frequency in teaching practice.

- The doubling rule does not necessarily denote a relationship of direct proportionality.

(3:20) The narrator presents a «trick» to find out whether two magnitudes are directly proportionate: «To find out if two magnitudes are directly proportionate, all you have to do is check that doubling one is the same as doubling the other, tripling is the same as tripling.»

This is a necessary but not sufficient condition for two magnitudes to be directly proportionate. The proportionality of magnitudes is a linear function established between the values of the magnitudes.

- When the relation of direct proportionality is justified, this is based on the relationship that doubling one magnitude corresponds to doubling the other.

(4:30) Next, the narrator tells the students the question they should ask after finding the magnitudes: «Will twice as many shirts need twice as much cloth?»

(4:43) «If the answer is yes, and in this video it obviously will be, because if not, we would be looking at another type of proportionality ... then we are looking at a problem of direct proportion.»

This is incorrect. The answer to a question like the one he asks might be no and it might be that no other situation of proportionality is involved.

- Neither the operations in a proportion nor the reason for using cross multiplication are explained.

We consider that the degree of suitability regarding relationships between objects, on a low-medium-high ordinal scale, is medium, as not all of the propositions and procedures have an associated argument. Furthermore, the various meanings of the objects that appear in the exercises are sometimes but not always identified, and so the degree of suitability is medium in this aspect.

GRAPH 2. Screenshot from minute 8:15. General method.

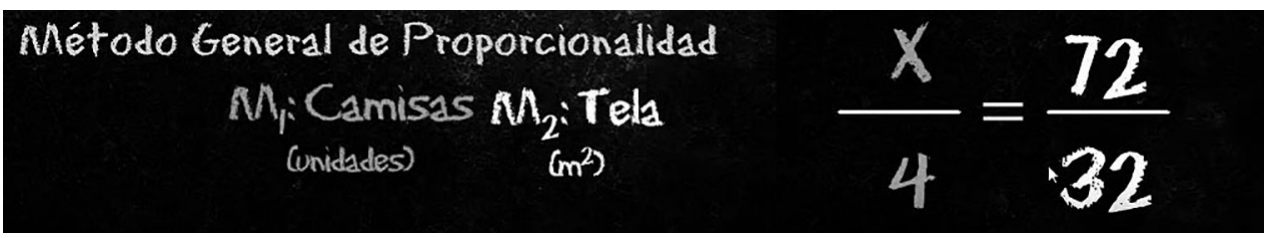

Image text: General Method of Proportionality. $M_{1}$ : shirts (units) $M_{2}$ : fabric $\left(m^{2}\right)$ Source: Clasemáticas, 2012. 
(8:15) «In other words, the quotients are equal between the magnitudes, within them, or comparing one with the other magnitude, so this quotient is also correct.»

In the first method he presents, the «equal quotients» one, he uses external ratios. Here, in what the author calls the "general proportionality method», he uses internal ratios, without appropriately explaining the relationship between the two proportions. Furthermore, he uses the term proportion instead of ratio to refer to the fractions that appear on screen.

The final procedure for solving proportionality problems that the author of the video presents is the rule of three:

(11:35) Put the magnitudes in a column; «we put $x$ is to 72 as 4 shirts is to 32 .»

GRAPH 3. Screenshot from minute 11:47. Rule of three method.

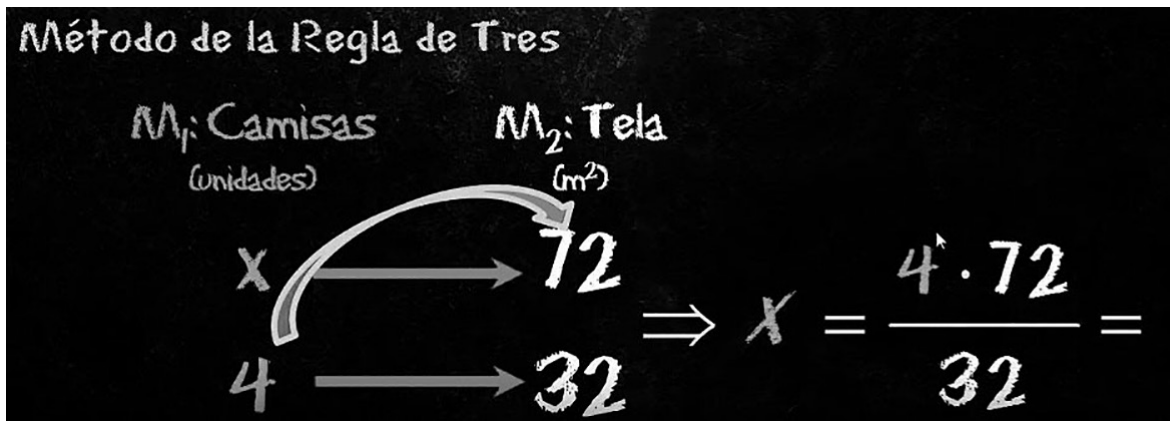

Image text: Rule of three method. $\mathrm{M}_{1}$ : shirts (units) $\mathrm{M}_{2}$ : fabric $\left(\mathrm{m}^{2}\right)$

Source: Clasemáticas, 2012.

(11:47) «And this, in reality, if you rewind the video, is the general method. The thing is I don't know why you love putting arrows on it. The general method has a mathematical explanation and this one doesn't really.»

He relates the rule of three method, which he solves by cross multiplication, to the general method. Thus, he is referring to to a "debased rule of three», that is to say, he does not present the proportional equation, which he distinguishes as another method. Therefore, what he calls "the rule of three» is, in the diagram (using arrows), accompanied by cross multiplication, which is not justified in the video.

Based on these analyses, the researchers quantitatively evaluated the video's degree of epistemic suitability in each of the six components, scoring each indicator according to whether its contribution to the suitability is low, medium, or high $(0,1$, or 2 points, respectively). The total maximum score will, therefore, be 12 . Table 1 shows the marks given by the research team. 
The issue of didactical suitability in mathematics educational videos: an analysis experiment...

TABLE 1. Evaluation of epistemic suitability by components.

\begin{tabular}{|c|c|}
\hline Component & Score given to the video \\
\hline Situations-problems & 1 \\
\hline Language & 2 \\
\hline Rules & 1 \\
\hline Arguments & 1 \\
\hline Relationships between objects & 1 \\
\hline Articulation of meanings & 1 \\
\hline Final score & 7 \\
\hline
\end{tabular}

Source: Own elaboration.

\section{Results}

For the second working session, we asked the students firstly to watch at home three videos ${ }^{2}$ about directly proportional distributions which had different degrees of suitability. Individually, they had to decide on the greater or lesser degree of suitability of the videos as well as their level of use of algebra, taking into account the type of solution developed. Afterwards, in class, they were asked to discuss their evaluations with the work group and then prepare a group opinion about the degree of suitability of the different videos. The instructions given to the students for analysing the videos are the same as the ones proposed in the final task. That is to say, should consider: a) the variety of situations-problems presented; b) the presence of different registers of representation; c) the clarity and correctness of the definitions, propositions, and procedures; and d) the argumentation of the propositions and procedures.
As a result of this session, we saw that the future teachers overlooked the errors in definitions, propositions or procedures present in the videos and that in the idea-sharing in the working group, they found it hard to agree on the degree of suitability of the different videos. Discussing the individual positions in many cases led them to modify their preliminary analyses, identifying new elements of analysis that had gone unnoticed.

In this section, we analyse the answers the students gave in the final evaluation task (third working session), which consequently reflect what these students have learnt. We then analyse the answers given to the quantitative evaluation instruction for each of the six components of suitability, as well as the overall adequacy of the video.

Table 2 shows the frequencies and percentages of the answers given regarding the characteristics of the situations-problems presented in the video. 
TABLE 2. Variety of situations-problems proposed $(n=93)$.

\begin{tabular}{|c|c|}
\hline Characteristics of the situations & Frequency (\%) \\
\hline A representative and defined sample of problems is presented & $83(89.25)$ \\
\hline The situations appear in context & $83(89.25)$ \\
\hline The mathematical ideas are connected & $87(93.55)$ \\
\hline Various ways of tackling the problems are suggested & $89(95.70)$ \\
\hline
\end{tabular}

Source: Own elaboration.

We can see that the majority of the students adequately recognised that the situations are presented in context in the educational video being analysed, the ideas are connected, and various ways of approaching the problems are provided. The majority of them (89.25\%) also accepted that the videos show a representative and defined sample of problems.
In relation to the presence of different representations and language registers, the students have no difficulty in identifying the natural register and the symbolic register. However, they identify the tabular and graphic registers to a lesser extent. Other students, to a lesser extent, identify animation and other different types (Table 3).

TABLE 3. Identification of linguistic representations ( $n=93)$.

\begin{tabular}{|c|c|}
\hline Type of language & Frequency (\%) \\
\hline Natural (oral) & $92(98.9)$ \\
\hline Natural (written) & $93(100.0)$ \\
\hline Symbolic (numerical) & $89(95.7)$ \\
\hline Symbolic (algebraic) & $83(89.2)$ \\
\hline Tabular & $74(79.6)$ \\
\hline Graphic & $28(30.1)$ \\
\hline Animation & $49(52.7)$ \\
\hline Others (iconic, multimedia, diagrammatic, etc.) & $10(10.8)$ \\
\hline
\end{tabular}

Source: Own elaboration.

The majority of the future teachers have difficulties locating the errors or inaccuracies in the definitions, propositions or procedures, as well as in the ar- guments given for the transformations, or when the relationship of direct proportionality in the situations presented is justified (Table 4). 
The issue of didactical suitability in mathematics educational videos: an analysis experiment...

TABLE 4. Clarity and correction of definitions, propositions, procedures, and arguments.

\begin{tabular}{|c|c|c|c|c|c|}
\hline \multirow{2}{*}{\multicolumn{2}{|c|}{$\begin{array}{l}\text { Errors and inaccuracies in the } \\
\text { rules and arguments }\end{array}$}} & \multicolumn{4}{|c|}{ Frequency (\%) } \\
\hline & & Not & Explicitly & $\begin{array}{l}\text { Yes but } \\
\text { does not }\end{array}$ & Yes and \\
\hline \multirow{3}{*}{ Rules } & $\begin{array}{l}\text { Errors in } \\
\text { arithmetic-algebraic } \\
\text { treatment }\end{array}$ & $63(67.7)$ & $21(22.6)$ & $2(2.2)$ & $7(7.5)$ \\
\hline & Errors in definitions & $51(54.8)$ & $22(23.7)$ & $2(2.2)$ & $18(19.4)$ \\
\hline & $\begin{array}{l}\text { Errors in proposi- } \\
\text { tions or procedures }\end{array}$ & $51(54.8)$ & $21(22.6)$ & $5(5.4)$ & $16(17.2)$ \\
\hline \multirow{3}{*}{ Arguments } & $\begin{array}{l}\text { Error or inaccuracy } \\
\text { when justifying a } \\
\text { relationship } \\
\text { of proportionality }\end{array}$ & $45(48.4)$ & $20(21.5)$ & $6(6.5)$ & $22(23.7)$ \\
\hline & $\begin{array}{l}\text { Error or inaccuracy } \\
\text { when justifying an } \\
\text { arithmetic-algebraic } \\
\text { transformation }\end{array}$ & $54(58.1)$ & $20(21.5)$ & $4(4.3)$ & $15(16.1)$ \\
\hline & $\begin{array}{l}\text { Other errors or } \\
\text { inaccuracies }\end{array}$ & $52(55.9)$ & $20(21.5)$ & $2(2.2)$ & $19(20.4)$ \\
\hline
\end{tabular}

Source: Own elaboration.

When the future teachers identify errors of arithmetic or algebraic processes, some mention that the author of the video does not explain some of the symbols used, including subscripts, or that simplifying the results of the operations might confuse the students.

With regards to errors or inaccuracies the students recognise in definitions, we find the following categories of answers:

- Error in the definition of ratio. This is the category with most answers that identify conflicts in the definitions.
For example, E50 said: «Ratio: the video defines this as the proportion of the quotient between both magnitudes. This could cause confusion; one alternative would be "link between two magnitudes that can be compared to each other."»

- Inaccuracy in the definition of direct proportionality.

- Confusing definitions or ones that are inappropriate for primary school.

- As well as these categories, some students include as errors in the defini- 
tions descriptions that are not relevant in relation to procedures or modes of expression. For example, E84 notes that: «The names used for the methods for calculating the proportion could cause confusion as in different places different names might be used for these methods.»

The future teachers show difficulties assigning errors to the appropriate categories and providing relevant descriptions. They refer to «confusing explanations» in «Errors relating to definitions». For example, E54 identifies as an error in definitions:

In the explanation of the proportionality method (in the exercise with the shirts) he isolates "X»" as if it were an equation. $\mathrm{He}$ should have found the proportion between the shirts and the metres of cloth (how many times bigger the metres of cloth are than the number of shirts) and then divided the metres of cloth to get « $\mathrm{x} »$.

Then adds regarding «Errors in propositions or procedures»: «In procedures, I would put the same error as in "Errors in definitions"».

Most of the students who identify conflicts in procedures refer to the rule of three: «He crosses out numbers and isolates « $\mathrm{X}$ » in the rule of three confusingly, writing small numbers next to big ones might give the impression they are powers $(\mathrm{E} 52) »$.

Other students identify procedural errors: "In the equal quotients method, putting the unknown quantity « $\mathrm{X}$ » in the numerator, it is easier for the student to place it in denominator of the left fraction (E26).»

Or they feel that the explanations that accompany the procedures are complex or insufficient.

Only one student (E83) refers to the error in the proposition: «To find out wether two magnitudes are directly proportionate or not, all you have to do is check that doubling one is the same as doubling the other, tripling is the same as tripling ...»

No student mentions as an inaccuracy the fact that the argumentation of the relationship of direct proportionality is only based on the proposition that «doubling one magnitude corresponds to doubling the other». Most of the students who place an error in this category do so incorrectly, either because they indicate «confusing explanation» or «limited argumentations» or because they refer to inaccuracies in the explanation of transformations and not in the relationship of proportionality.

Only six students make any reference to the lack of argumentation of the operations. For example, E67 states that: "The only problem I can see with this video is that when simplifying he does not explain this process, it is true that the video is not about simplification but it could mean students get lost when solving problems.»

In relation to other errors, the future teachers fundamentally identify: 
The issue of didactical suitability in mathematics educational videos: an analysis experiment...

- Errors or inaccuracies in the expression (oral) or language used.

- Unsuitable presentation for primaryschool pupils.

- Difficulties understanding the differences between the methods for solving the problem.

- Insufficient arguments for arithmetic transformations.

Regarding the «trick» the author of the video presents, E68 includes the following as an error:

Trick: «To find out whether two magnitudes are directly proportional, it is enough to establish that the double of one of them corresponds to the double of the other». Although this is a quick way of see-

ing if there is direct proportionality or not, it seems imprecise as it only mentions doubling. He should mention «halves match", «sums match», «differences match», in other words, using all four operations.

Table 5 shows the frequency of errors detected by the future teachers. We distinguish between answers that mention an error in a somewhat relevant way in the appropriate category and answers that are not relevant because they are not in the appropriate category, because the description of the error is not conclusive or is subjective, or because what they include cannot be regarded as an error or inaccuracy. In general, the percentages of students who recognised errors and inaccuracies were low. The highest related to the justification of the relationship of proportionality, mentioned by 22 students $(23.7 \%)$.

TABLE 5. Errors identified by the students.

\begin{tabular}{|c|c|c|c|c|c|c|}
\hline \multirow{3}{*}{\multicolumn{2}{|c|}{$\begin{array}{l}\text { Errors and inaccuracies in the } \\
\text { rules and arguments detected by } \\
\text { the future teachers }\end{array}$}} & \multicolumn{5}{|c|}{ Frequency } \\
\hline & & \multirow[b]{2}{*}{$\begin{array}{l}\text { Relevant } \\
\text { answer }\end{array}$} & \multicolumn{3}{|c|}{ Irrelevant answer } & \multirow[b]{2}{*}{ Total $(\%)$} \\
\hline & & & $\begin{array}{l}\text { It is an } \\
\text { error but } \\
\text { not in the }\end{array}$ & $\begin{array}{l}\text { Inconclu- } \\
\text { sive or } \\
\text { subiective }\end{array}$ & Not & \\
\hline \multirow{3}{*}{ In rules } & $\begin{array}{l}\text { Arithmetic-algebraic } \\
\text { treatment }\end{array}$ & 1 & 1 & 1 & 4 & $7(7.5)$ \\
\hline & In definitions & 7 & 3 & 5 & 3 & $18(19.4)$ \\
\hline & In propositions-proportions & 4 & 3 & 5 & 4 & $16(17.2)$ \\
\hline \multirow{3}{*}{$\begin{array}{l}\text { In } \\
\text { argu- } \\
\text { ments }\end{array}$} & $\begin{array}{l}\text { When argumenting the rela- } \\
\text { tionship of proportionality }\end{array}$ & 3 & 8 & 4 & 7 & $22(23.7)$ \\
\hline & $\begin{array}{l}\text { When argumenting } \\
\text { transformation }\end{array}$ & 5 & 3 & 3 & 4 & $15(16.1)$ \\
\hline & Others & 5 & 2 & 7 & 5 & $19(20.4)$ \\
\hline
\end{tabular}

Source: Own elaboration. 
Section d) of the instructions asked the trainee teachers to identify whether the mathematical objects and the meanings are presented in connection in the video. Apart from two students who did not answer this section, $73.12 \%$ of the future teachers believed that the mathematical objects are related in a relevant way and that, as such, the degree of suitability of this aspect is high (Table 6). Furthermore, $63.44 \%$ said that the different meanings of the objects involved are always connected.

TABLE 6. Relationships between objects and meanings.

\begin{tabular}{|c|c|c|c|}
\hline \multicolumn{2}{|c|}{ Relationships between mathematical objects } & Suitability & Frequency (\%) \\
\hline \multirow{3}{*}{$\begin{array}{l}\text { Relationships between } \\
\text { objects }\end{array}$} & $\begin{array}{l}\text { All of the propositions } \\
\text { and procedures have an } \\
\text { associated argument }\end{array}$ & High & $68(73.1)$ \\
\hline & $\begin{array}{l}\text { Some of the propositions } \\
\text { and procedures have an } \\
\text { associated argument }\end{array}$ & Medium & $23(24.7)$ \\
\hline & $\begin{array}{l}\text { None of the propositions } \\
\text { and procedures have an } \\
\text { associated argument }\end{array}$ & Low & $0(0.0)$ \\
\hline \multirow{3}{*}{$\begin{array}{l}\text { The meanings of the } \\
\text { objects that appear are } \\
\text { identified and interwoven }\end{array}$} & Always & High & $59(63.4)$ \\
\hline & Sometimes & Medium & $30(32.3)$ \\
\hline & Never & Low & $2(2.2)$ \\
\hline
\end{tabular}

Source: Own elaboration.

According to the results obtained in the jects and meanings), the future teachers preliminary analyses (variety and repre- had to quantitatively evaluate the degree sentativeness of the situations-problems of didactical suitability of the video. They proposed, variety of representation sys- had to assign a score of 0,1 , or 2 depending tems, clarity and correctness of the rules on whether they regarded its suitability as and arguments, connection between ob- low, medium, or high, respectively.

TABLE 7. Frequency (\%) of evaluation of epistemic suitability by components.

\begin{tabular}{|l|c|c|c|}
\hline \multirow{2}{*}{ Components } & \multicolumn{3}{|c|}{ Evaluation of the video } \\
\cline { 2 - 4 } & $\mathbf{0}$ & $\mathbf{1}$ & $\mathbf{2}$ \\
\hline Situations-problems & $1(1.1)$ & $40(43.0)$ & $52(52.9)$ \\
\hline Language & $3(3.2)$ & $37(39.8)$ & $53(57.0)$ \\
\hline Rules & $3(3.2)$ & $33(35.5)$ & $57(61.3)$ \\
\hline Arguments & $1(1.1)$ & $42(45.2)$ & $50(53.8)$ \\
\hline Relationships between objects & $4(4.3)$ & $22(23.7)$ & $67(72.0)$ \\
\hline Articulation of meanings & $2(2.2)$ & $37(39.8)$ & $54(58.1)$ \\
\hline
\end{tabular}

Source: Own elaboration. 
The issue of didactical suitability in mathematics educational videos: an analysis experiment...

Table 7 shows that over half of the students gave the highest mark for relevance in each of the epistemic suitability components. The aspect with the highest valuation is the relationship between objects (72.0\% indicated high suitability here) followed by correctness of rules $(61.3 \%)$.

We can see that the highest score that can be assigned to the video is 12 points. The minimum score assigned was 4 points (one student) and the mean was
9.5 points. The most common score (in $29 \%$ of cases) was 10 points. Furthermore, 9 students (10\%) gave the video the maximum score.

The future teachers were asked to explain the reasons for their epistemic suitability scores. Analysing their answers has enabled us to identify the categories in Tables 8 and 9, where we distinguish arguments for a positive evaluation and arguments for a negative evaluation.

TABLE 8. Arguments for giving a positive evaluation $(n=93)$.

\begin{tabular}{|l|c|}
\hline Indicator & Frequency (\%) \\
\hline Attractive presentation & $8(8.6)$ \\
\hline Adequate language & $43(46.2)$ \\
\hline Varied language & $21(22.6)$ \\
\hline $\begin{array}{l}\text { Variety of examples (representative and articulated } \\
\text { sample of situations-problems) }\end{array}$ & $26(28.0)$ \\
\hline Everyday situations-context/connection with real life & $27(29.0)$ \\
\hline Appropriate arguments/clear explanations & $38(40.9)$ \\
\hline Enough arguments & $17(18.3)$ \\
\hline Varied and adequate procedures/methods & $23(24.7)$ \\
\hline Coherent/adequate definitions & $19(20.4)$ \\
\hline Articulated meanings & $17(18.3)$ \\
\hline $\begin{array}{l}\text { Favours reasoning or that pupils construct, perfect, and } \\
\text { use their own representations to organise, record, and } \\
\text { share ideas }\end{array}$ & $14(15.1)$ \\
\hline $\begin{array}{l}\text { Appropriate relationships between objects (propositions } \\
\text { with associated argument; related methods) }\end{array}$ & $15(16.1)$ \\
\hline No error in rules or arguments & $7(7.5)$ \\
\hline
\end{tabular}

Source: Own elaboration.

The future teachers gave a positive valuation to the language used and to the arguments used being appropriate and the explanations clear. For example, E14 gave 2 points to the language component, saying:
The language used is varied: natural (oral and written), symbolic (numerical and algebraic), tabular (using tables), animation (a child thinking and asking a question, a gif with movement in the result of the problem), and with arrows establishing relationships of proportionality. 
... Also, the level of language is clear and simple suitable for the Primary Education level it is aimed at.

E22 gives the arguments component 2 points: «I did not think there were any notable errors regarding the arguments and the procedures used in the video. The arithmetic operations are always accompanied by an argument and justification. The arguments and procedures are clear.»
However, these components are where negative evaluations are most often given, referring both to errors in expression and language and to unclear or confusing arguments (Table 9). E38 said: «Sometimes it uses language that is a bit technical for a primary-school child. I think the arguments are good, but they could be a bit clearer in some of the 4 methods it explains for direct proportionality.»

TABLE 9. Arguments for giving a negative evaluation $(n=93)$.

\begin{tabular}{|l|c|}
\hline Indicator & Frequency (\%) \\
\hline Situations-problems not adequate for primary level & $3(3.2)$ \\
\hline Errors in expression/language & $11(11.8)$ \\
\hline Language not suitable for primary level & $11(11.8)$ \\
\hline $\begin{array}{l}\text { Poor representations (little connection, lack of graphic or visual lan- } \\
\text { guage) }\end{array}$ & $3(3.2)$ \\
\hline Difficulty distinguishing methods & $4(4.3)$ \\
\hline Excessive arguments & $3(3.2)$ \\
\hline Unclear, confusing or fast arguments & $19(20.4)$ \\
\hline Propositions with no connected argument & $1(1.1)$ \\
\hline Incorrect or insufficient definitions & $2(2.2)$ \\
\hline Definitions not adequate for primary level & $4(4.3)$ \\
\hline Procedures not adequate for primary level & $3(3.2)$ \\
\hline Procedures unclear or fast & $4(4.3)$ \\
\hline Errors in procedures (simplification in rule of 3) & $5(5.4)$ \\
\hline Arguments not adequate for primary level & $4(4.3)$ \\
\hline Unconnected meanings & $12(12.9)$ \\
\hline Lacks situations of meaning construction or own representations & $3(3.2)$ \\
\hline She author includes ideas out of context or that might cause confusion & $3(3.2)$ \\
\hline for example, inverse/compound proportionality) & $4(4.3)$ \\
\hline Situations contextualised poorly or only in a mathematical context & 3 \\
\hline
\end{tabular}

Source: Own elaboration. 
The issue of didactical suitability in mathematics educational videos: an analysis experiment...

The analysis as a whole should lead fu- students' responses into «yes», «yes, but ture teachers to decide whether the video ...» when they make some kind of objection, seems suitable to them, explaining their and «no». Table 10 includes the frequencies decision. In this case, we have classified the and percentages of each of these options.

TABLE 10. Adequacy of the video in the final reflection $(n=93)$.

\begin{tabular}{|l|c|}
\hline In your opinion, is this video adequate? & Frequency (\%) \\
\hline Yes & $48(51.61)$ \\
\hline Yes but... & $38(40.86)$ \\
\hline No & $7(7.53)$ \\
\hline
\end{tabular}

Source: Own elaboration.

Of the future teachers, $51.61 \%$ consider adequacy of the argumentation. Table 11 the video to be adequate, and they valued summarise the different explanations the most highly the presence of various ways students used to evaluate how adequate of solving the problems and the degree of the video is.

TABLE 11. Arguments when they believe the video is totally adequate $(\mathrm{n}=48)$.

\begin{tabular}{|l|c|}
\hline Indicator & Frequency (\%) \\
\hline Various ways of solving problems & $46(95.8)$ \\
\hline Variety of examples & $22(45.8)$ \\
\hline Adequate and detailed explanation & $42(87.5)$ \\
\hline $\begin{array}{l}\text { Simplifies definitions/presents the most relevant and representative } \\
\text { information }\end{array}$ & $13(27.1)$ \\
\hline Adequate representation/language & $26(54.2)$ \\
\hline Useful/motivational & $7(14.6)$ \\
\hline Includes tricks/advice & $12(25.0)$ \\
\hline Relatable context & $10(10.8)$ \\
\hline Inspires reflection on most adequate method & $6(12.5)$ \\
\hline
\end{tabular}




\begin{tabular}{|l|c|}
\hline There are no errors & $7(14.6)$ \\
\hline Adequate length & $2(4.2)$ \\
\hline Attractive presentation, informal tone & $5(10.4)$ \\
\hline
\end{tabular}

Source: Own elaboration.

Of the future teachers, $40.86 \%$ did not methods used being inadequate for pribelieve that the video was wholly adequa- mary-level, or it potentially being boring te (Table 10). They identify as drawbacks for the pupils.

the length, the language, and some of the

TABLE 12. Arguments that indicate drawbacks («yes but ...») $(\mathrm{n}=38)$.

\begin{tabular}{|c|c|}
\hline Indicator & Frequency $(\%)$ \\
\hline Language not adequate for primary level & $11(28.9)$ \\
\hline Arithmetic procedures not explained & $4(10.5)$ \\
\hline Excess length & $6(15.8)$ \\
\hline $\begin{array}{l}\text { Boring or causes pupils to switch off, lacks animations or } \\
\text { visual resources }\end{array}$ & $19(50)$ \\
\hline Causes confusion among the students & $6(15.8)$ \\
\hline Lacks variety of contexts & $3(7.9)$ \\
\hline Incorrect explanations & $5(13.2)$ \\
\hline Too long and dense for primary school & $11(28.9)$ \\
\hline $\begin{array}{l}\text { Not all situations/concepts/methods are adequate } \\
\text { for primary level }\end{array}$ & $12(31.6)$ \\
\hline $\begin{array}{l}\text { Does not take into account prior knowledge or problems } \\
\text { with learning }\end{array}$ & $4(10.5)$ \\
\hline Contains errors & $7(18.4)$ \\
\hline
\end{tabular}

Source: Own elaboration. 
Finally, only $7.5 \%$ of the students did not believe the video was adequate. They state that the explanations are not very accurate or not at all accurate and the arguments are inadequate or confusing for primary level.

Analysing students' answers has enabled us to detect some conflicts relating to the identification of components and descriptors in the analysis of epistemic suitability, essentially relating to rules and articulation of meanings. For example, E32 identifies rules with ways of solving a problem and states: «I have given the rules a mark of 2 because they do display a wide variety of ways of solving the problem.»

Similarly, E56 noted that:

For the rules, I have given a score of 2 (high) for suitability because I agree with how he does the operations in each case where he solves the problems and because I do not think he makes any errors when doing them.

Some students consider that the meanings are articulated when various methods are used or what is being done in the video is justified. For example, E41 said: «I think that the definition of concepts has a high level, because it uses various methods to solving the problems.»

E30 added: «Regarding the articulation of meanings, the suitability is high as it constantly explains what each thing being done means.»

\section{Summary, implications, and li- mitations}

The aim of this work was to design, implement, and evaluate a training activity for future primary school teachers focussed on developing the knowledge and competence to analyse the epistemic suitability of educational videos on proportionality. We started by stating why the topic is of interest, given the abundance and availability of videos that are offered as resources to help teach mathematics. However, given the inconsistent quality and variety of meanings in the educational videos (Beltrán-Pellicer, Giacomone, \& Burgos, 2018), there is a need to train teachers in how to evaluate these resources and use them suitably.

The training activity focusses on providing future teachers with a theorybased tool for analysing epistemic suitability, that is to say, the mathematical content presented in a video about proportionality. Preliminary analysis by the researchers of the video revealed errors and inaccuracies in definitions, propositions, and procedures, as well as shortcomings or inaccuracies in the explanations used to justify the procedures and propositions. Also, the presentation and treatment of a variety of situationsproblems and the articulation of meanings of proportionality have major shortcomings, and so we rated its level of epistemic suitability as medium on the low-medium-high ordinal scale. However, the majority of the future teachers, after the training process had been applied, ra- 
ted the degree of suitability of the video as high in almost all components.

The evaluation instrument used, based on epistemic suitability components and indicators, considers the variety of situations-problems posed, the presence of different registers of representation, the clarity and correctness of the definitions, propositions, and procedures, and the justification for procedures and propositions using arguments that are relevant and adapted to the corresponding educational level. Failure to recognise the absence of some important indicators led a high percentage of students (51.6\%) to regard the video as an adequate teaching resource without identifying its shortcomings.

We also observed a degree of inconsistency in the allocation of scores by the participants, finding some evaluations with the maximum score where students identify more drawbacks than in others with lower scores. While these variations keep the overall evaluation coherent, they do indicate that it is perhaps necessary to define the criteria more, in order to achieve greater uniformity between participants. Nonetheless, we have to take into account that this is a qualitative evaluation of suitability and that the final number, within certain margins, is only the summary result of a more complex process in which the different components of suitability are identified.

These results suggest there is a need to consider in greater depth the development of future teachers' specialised con- tent knowledge, in this case regarding proportionality, increasing the training time allocated, analysing a larger variety of educational videos, and increasing group discussion of the results of the analysis phase.

This type of training action, focussing on the content of the discipline but with a clear orientation towards knowledge and didactic competences, aligns with works by other authors such as Davis (2015), where he notes the impact of his concept study, both when revealing the complexity of the underlying mathematical ideas and when developing the mathematical ideas necessary for teaching.

Furthermore, in addition to the epistemic element, the didactical suitability theoretical tool includes the cognitive, emotional, interactional, media, and ecological aspects, which are not considered in this piece of research. Although it is not relevant to apply some of these aspects to the case of using didactic resources, in particular the aspect of learning achieved, the other aspects could be the subject of analysis and reflection by the teachers who use these resources.

\section{Notes}

1 The video from the Clasemáticas channel can be viewed at https://www.youtube.com/watch?$\mathrm{v}=01 \mathrm{Mu} \cdot \mathrm{kgv} \cdot \mathrm{o}$

2 The videos suggested can be viewed at: https://www.youtube.com/watch?v=0Z5DejetHR8 https://www.youtube.com/watch?v=v8KN44iNPIs https://www.youtube.com/watch?v=1uAblb-McLo 


\section{References}

Aroza, C. J., Godino, J. D., \& Beltrán-Pellicer, P. (2016). Iniciación a la innovación e investigación educativa mediante el análisis de la idoneidad didáctica de una experiencia de enseñanza sobre proporcionalidad. Avances en Investigación e Innovación - Revista de Educación Secundaria, 6 (1), 1-29.

Azer, S. A., AlGrain, H. A., AlKhelaif, R. A., \& AlEshaiwi, S. M. (2013). Evaluation of the Educational Value of YouTube Videos about Physical Examination of the Cardiovascular and Respiratory Systems. Journal of Medical Internet Research, 15 (11), e241. doi:10.2196/jmir.2728

Bartell, T. G., Webel, C., Bowen, B., \& Dyson, N. (2013). Prospective teacher learning: recognizing evidence of conceptual understanding. Journal of Mathematics Teacher Education, 16 (1), 57-79.

Beltrán-Pellicer, P., Giacomone, B., \& Burgos, M. (2018). Online educational videos according to specific didactics: the case of mathematics. $\mathrm{Cul}$ tura y Educación, 30 (4), 633-662.

Beltrán-Pellicer, P., Godino, J. D., \& Giacomone, B. (2018). Elaboración de indicadores específicos de idoneidad didáctica en probabilidad: aplicación para la reflexión sobre la práctica docente. Bolema, 32 (61), 526-548

Ben-Chaim, D., Keret, Y., \& Ilany, B. (2012) Ratio and proportion: Research and teaching in mathematics teachers' education. Rotterdam: Sense Publisher.

Bergmann, J., \& Sams, A. (2012). Flip your classroom. Eugene, Oregon: International Society for Technology in Education.

Berk, D., Taber, S. B., Gorowara, C. C., \& Petzl, C. (2009). Developing prospective elementary teachers' flexibility in the domain of proportional reasoning. Mathematical Thinking and Learning, 11 (3), 113-135.

Borba, M. C., Askar, P., Engelbrecht, J., Gadanidis, G., Llinares, S., \& Aguilar, M. S. (2016). Blended learning, e-learning and mobile learning in mathematics education. ZDM. Mathematics Education, 48 (5), 589-610.
Breda, A., Pino-Fan, L., \& Font, V. (2017). Meta didactic-mathematical knowledge of teachers: criteria for the reflections and assessment on teaching practice. Eurasia Journal of Mathematics, Science \& Technology Education, 13 (6), 1893-1918.

Chapman, 0. (2014). Overall commentary: understanding and changing mathematics teachers. In J. J. Lo, K. R. Leatham, \& L. R. Van Zoest (Eds.), Research Trends in Mathematics Teacher Education (pp. 295-309). Dordrecht: Springer International Publishing.

Clasemáticas Canal (2012, November 8). (Proporcionalidad) - Proporción Directa (1002) [Video file]. Retrieved from https://www.youtube.com/ watch? $\mathrm{v}=01 \mathrm{Mu}-\mathrm{lkgv}-0$ (Consulted on 2019-0406).

Dabbagh, B., \& Kitsantas, A. (2012). Personal Learning Environments, social media, and self-regulated learning: A natural formula for connecting formal and informal learning. The Internet and Higher Education, 12 (1), 3-8.

Daniel Carreon (2017, June 27). REPARTO PROPORCIONAL-Super fácil [Video file]. Retrieved from https://www.youtube.com/ watch?v=1uAbIb-McLo (Consulted on 201904-06).

Davis, B. (2015). The mathematics that secondary teachers (need to) know. revista española de pedagogía, 73 (261), 321-342.

Duffy, P. (2008). Engaging the YouTube Google-eyed generation: Strategies for using Web 2.0 in teaching and learning. Electronic Journal of E-learning, 6 (2), 119-130.

English, L. D. (2008). Setting an agenda for international research in mathematics education. In L. D. English \& D. Kirshner (Eds.), Handbook of international research in mathematics education (pp. 3-19). New York \& London: Taylor and Francis (Routledge).

Godino, J. D. (2013). Indicadores de idoneidad didáctica de procesos de enseñanza y aprendizaje de las matemáticas. Cuadernos de Investigación y Formación en Educación Matemática, 11, 111-132. 
Godino, J. D., Batanero, C., \& Font, V. (2007). The onto-semiotic approach to research in mathematics education. ZDM. Mathematics Education, 39 (1-2), 127-135.

Godino, J. D., Font, V., Wilhelmi, M. R., \& Lurduy, 0. (2011). Why is the learning of elementary arithmetic concepts difficult? Semiotic tools for understanding the nature of mathematical objects. Educational Studies in Mathematics, 77 (2), 247-265.

Godino, J. D., Giacomone, B., Batanero, C., \& Font, V. (2017). Enfoque ontosemiótico de los conocimientos y competencias del profesor de matemáticas. Bolema, 31 (57), 90-113.

Hilton, A., \& Hilton, G. (2018). Primary school teachers implementing structured mathematics interventions to promote their mathematics knowledge for teaching proportional reasoning. Journal of Mathematics Teacher Education, 22 (6), 545-574. doi: https://doi.org/10.1007/ s10857-018-9405-7

Mason, J. (2016). Perception, interpretation and decision making: understanding gaps between competence and performance-a commentary. ZDM. Mathematics Education, 48 (1-2), 219226.

Pino-Fan, L., \& Godino, J. D. (2015). Perspectiva ampliada del conocimiento didáctico-matemático del profesor. Paradigma, 36 (1), 87-109.

Ponte, J. P., \& Chapman, O. (2016). Prospective mathematics teachers' learning and knowledge for teaching. In L. D. English \& D. Kirshner (Eds.), Handbook of international research in mathematics education (pp. 275-296). New York: Routledge.

Portugal, K. O., Arruda, S. D. M., \& Passos, M. M. (2018). Free-choice teaching: how YouTube presents a new kind of teacher. Revista Electrónica de Enseñanza de las Ciencias, 17 (1), 183-199.

Posadas, P., \& Godino, J. D. (2017). Reflexión sobre la práctica docente como estrategia formativa para desarrollar el conocimiento didáctico-matemático. Didacticae, 1, 77-96. doi: https://doi. org/10.1344/did.2017.1.77-96

Ramírez, A. (2010). Youtube y el desarrollo de la competencia matemática. Resultados de una investigación cuasi-experimental. Contextos Educativos, 13, 123-138.
Ruiz-Reyes, K., Contreras, J. M., Arteaga, P., \& Oviedo, K. (2017). Análisis semiótico de videos tutoriales para la enseñanza de la probabilidad en educación primaria. In J. M. Contreras, P. Arteaga, G. R. Cañadas, M. M. Gea, B. Giacomone, \& M. M. López-Martín (Eds.), Actas del Segundo Congreso International Virtual sobre el Enfoque Ontosemiótico del Conocimiento y la Instrucción Matemáticos. Retrieved from http://hdl.handle.net/10481/45382 (Consulted on 2019-07-19).

Sadler, D. R. (2013). Making competent judgments of competence. In S. Blömeke, 0. ZlatkinTroitschanskaia, C. Kuhn, \& J. Fege (Eds.), Modeling and measuring competencies in higher education: Tasks and challenges (pp. 1327). Rotterdam: Sense Publishing.

Santos, J. A. (2018). Valoración de vídeo tutoriales de matemáticas disponibles en internet. Nuevos instrumentos para el análisis de los procesos educativos (Doctoral dissertation). Centro de Investigación y de Estudios Avanzados del Instituto Politécnico Nacional, Ciudad de México, Mexico.

Smatematico (2013, April 7). Problema de reparto proporcional [Video file]. Retrieved from https://www.youtube.com/watch?v=0Z5DejetHR8 (Consulted on 2019-04-06).

Turney, C. S. M., Robinson, D., Lee, M., \& Soutar, A. (2009). Using technology to direct learning in higher education. The way forward? Active Learning in Higher Education, 10 (1), 71-83.

Tuto mate (2016, May 10). Problemas de repartos directamente proporcionales [Video file]. Retrieved from https://www.youtube.com/watch?$\mathrm{v}=\mathrm{v} 8 \mathrm{KN} 44$ iNPls (Consulted on 2019-04-06).

\section{Authors' biographies:}

Maria Burgos is an Associate Lecturer in the Department of Didactic of Mathematics at the Universidad de Granada. She has a doctorate in Mathematics from the Universidad de Almería and a master's in Didactic of Mathematics from the Universidad de Granada. She is a member of the FQM-126 Teoría de la Educación Matemática y Educación Estadística (Theory 
of Mathematics Education and Statistics Education) group.

iD https://orcid.org/0000-0002-4598-7684

Pablo Beltrán-Pellicer is a Associate Lecturer in the Didactic of Mathematics area of the Universidad de Zaragoza and a Compulsory Secondary Education and Spanish Baccalaureate Teacher at the CPI (Integrated Public School) Val de la Atalaya (María de Huerva, Zaragoza). He has a doctorate in Mathematics Teaching and is a member of the S36_17D Investigación en Educación Matemática (Mathematicas Education Research) group.

(iD https://orcid.org/0000-0002-1275-9976
Juan D. Godino is a retired Professor of Didactics of Mathematics, Extraordinary Collaborator of the Department ofDidactics of Mathematics and professor of the Doctoral Program in Education of the University of Granada. Member of the FQM-126 Group, Theory of Mathematical Education and Statistical Education. Since 1993 he has been developing the onto-semiotic approach to mathematical knowledge and instruction, a specific theoretical framework for mathematical education.

iD http://orcid.org/0000-0001-8409-0258 


\section{revista española de pedagogía año 78, n 275, enero-abril 2020 \\ Spanish Journal of Pedagogy \\ year 78, n. 275, January-April 2020 \\ Table of Contents \\ Sumario}

\section{Mathematics teaching} issues

Cuestiones de enseñanza de las matemáticas

Wendolyn Elizabeth Aguilar-Salinas, Maximiliano de las Fuentes-Lara, Araceli Celina Justo-López, \& Ana Dolores Martínez-Molina

A measurement instrument for establishing the algebraic skills of engineering students on a Differential Calculus Course in engineering Instrumento de medición para diagnosticar las habilidades algebraicas de los estudiantes en el Curso de Cálculo Diferencial en ingeniería

Maria Burgos, Pablo Beltrán-Pellicer, \& Juan D. Godino The issue of didactical suitability in mathematics educational videos: experience of analysis with prospective primary school teachers La cuestión de la idoneidad de los vídeos educativos de matemáticas: una experiencia de análisis con futuros maestros de educación primaria

27
Online training in the world of education: experiences from the United States La formación online en el mundo de la educación: experiencias de los Estados Unidos

Gerald LeTendre, \& Tiffany Squires

Integrating online and residential master's programs in education

Integración de programas de máster online y presenciales en educación

\section{Laurence B. Boggess}

Innovations in online faculty development: an organizational model for long.term support of online faculty

Innovación en la capacitación docente online: un modelo organizacional para brindar apoyo a largo plazo a la docencia online

Brian Redmond

Shared team leadership for an online program Liderazgo de equipo compartido de un programa online 
Studies and Notes

Estudios y Notas

\section{Paolo Scotton}

Thinking together, living fully. Experiencing philosophy

with children

Pensar en común, vivir en plenitud. La experiencia de la

filosofía con los niños y las niñas

103

Manuel Delgado-Garcia, Sara Conde Vélez, \& Ángel

\section{Boza Carreño}

Profiles and functions of university tutors and their

effects on students' tutorial needs

Perfiles y funciones del tutor universitario y sus efectos sobre

las necesidades tutoriales del alumnado

119
Book reviews

Luri, G. (2019). La imaginación conservadora: una defensa apasionada de las ideas que han hecho del mundo un lugar mejor [The conservative imagination: A passionate defence of ideas that have made the world a better place] (Enrique Alonso Sainz). Quigley, C. F., \& Herro, D. (2019). An educator's guide to STEAM. Engaging students using real-world problems (Juan Luis Fuentes). Prince, T. (2019). Ejercicios de mindfulness en el aula. 100 ideas prácticas [Mindfulness exercises for the class. 100 practical ideas] (José V. Merino Fernández).

145

\section{Call for papers}

Solicitud de originales

This is the English version of the research articles and book reviews published originally in the Spanish printed version of issue 275 of the revista española de pedagogía. The full Spanish version of this issue can also be found on the journal's website http://revistadepedagogia.org.

ISSN: 0034-9461 (Print), 2174-0909 (Online)

https://revistadepedagogia.org/

Depósito legal: M. 6.020 - 1958

INDUSTRIA GRÁFICA ANZOS, S.L. Fuenlabrada - Madrid 